\title{
Your Network or Mine? The Economics of Routing Rules
}

\author{
Benjamin E. Hermalin and Michael L. Katz
}

FEBRUARY 2004

\begin{abstract}
In many markets, including payment cards, telecommunications, and on-line auctions, service providers operate networks that support customer transactions with each other. When the two sides of a transaction belong to more than one network in common, the question of which network will carry the transaction arises. We show that the answer depends on a combination of who has the formal authority to choose and the parties' network subscription decisions. Our central finding is that granting formal authority to one side of the market can increase the extent to which transactions run over the network preferred by the other side of the market. We also characterize competing networks' equilibrium choices of routing rules and prices.
\end{abstract}

The authors wish to thank John Morgan, Patrick Rey, Jean-Charles Rochet, and seminar participants in Berkeley and Toulouse for helpful comments. Financial support from the Willis H. Booth Chair in Banking \& Finance and the Sarin Chair in Strategy \& Leadership is gratefully acknowledged. 


\section{Contents}

1 Introduction $\quad 1$

2 A Model $\quad 5$

3 The Connection Continuation Game $\quad 7$

3.1 One-Side-Chooses Routing . . . . . . . . . . . . . . . . . 8

3.2 Network Routing . . . . . . . . . . . . . . . . . . . . . . . . . . . . . . . . . . . . . . . .

3.3 One-Sided Exclusivity . . . . . . . . . . . . . . . . . 21

4 Network Pricing $\quad 24$

5 The Choice of Routing Regime $\quad 27$

6 Extensions $\quad 32$

6.1 Video Games and Operating Systems . . . . . . . . . . . . . . . . . 32

6.2 The Deciding Party Pays . . . . . . . . . . . . . . . . . . . . . 33

6.3 A Hotelling Model . . . . . . . . . . . . . . . . . . . . . . 34

6.4 Rent Dissipation in the Absence of Product Differentiation . . . . . . . . . 38

$\begin{array}{lll}7 & \text { Conclusion } & 41\end{array}$ 


\section{Introduction}

In many markets, service providers operate networks that support the interactions of their customers. Examples include payment systems, telecommunications networks, on-line auction sites, B2B electronic commerce exchanges, advertiser-supported media, and social clubs. ${ }^{1}$ Absent network interconnection and interoperability, two users must belong to the same network in order to transact with one another. In many settings, however, a single user can participate in multiple networks. When two users wish to transact with one another and they participate in multiple networks in common, the question arises, who decides which network carries the transaction? The answer is the subject of this paper. ${ }^{2}$

An example helps fix ideas. A credit or debit card network can be thought of as facilitating transactions between retail merchants on one side and and card-issuing banks or card-holding consumers on the other. ${ }^{3}$ A notable feature of the credit and debit card industries in many countries is that a typical merchant accepts cards that run over several different networks and a typical consumer holds a portfolio of cards that are collectively capable of running over multiple networks. Indeed, in the United States, at least, many financial institutions issue debit cards where each card is capable of running over multiple networks. Consequently, when a consumer presents his or her debit card to a merchant to make a purchase, it often

\footnotetext{
${ }^{1}$ Settings in which suppliers match two sides of a transaction have become known as two-sided markets. There has been considerable work on two-sided markets in recent years. Notable examples include Armstrong (2004), Caillaud and Jullien (2001, 2003), Doyle and Smith (1998), Hahn (2003), Hermalin and Katz (2004), Jeon et al. (2003), Kim and Lim (2001), Laffont et al. (2003), Rochet and Tirole (2003, 2004), Squire (1973), and Srinagesh and Gong (1996). Papers specifically considering the two-sided-market aspects of card payment systems include Schmalensee (2002), Tieman and Bolt (2003), Guthrie and Wright (2003), and the papers discussed by Rochet (2003) in his survey.

${ }^{2}$ Like us, Caillaud and Jullien (2001, 2003) and Rochet and Tirole (2003) consider competition between networks. These papers do not, however, focus on the question of routing rules, as they each assume a specific routing rule, which is taken as fixed exogenously. Our focus, instead, is on the endogenous determination of routing rules. Moreover, Caillaud and Jullien and Rochet and Tirole's papers assume the members of one or both sides of the market have homogenous preferences vis-à-vis the networks. In contrast, much of our attention is on situations in which the members of each side have heterogenous preferences vis-à-vis the networks.

${ }^{3}$ Actual debit networks are somewhat more complicated. Financial institutions that issue cards and acquire transactions for merchants are the actual members of a debit network. Cardholders are customers of the card-issuing banks, while merchants are customers of the acquiring banks. Moreover, physical routing decisions may be made by merchant processors, who handle the mechanics of routing transactions. Our simplified setting captures the important economic forces that underlie the routing issue.
} 
is possible to complete a given transaction over several different debit networks. This raises the issue of who chooses the specific network used. ${ }^{4}$

The answer depends, in part, on the technology used to authenticate the cardholder at the time of the transaction. The leading authentication methods today are signature and personal identification number (PIN). It is estimated that between 65 and 80 percent of debit cards are both PIN and signature enabled, ${ }^{5}$ and the choice between modes of authentication is typically made by the consumer, subject to incentives provided by merchants and card issuers. If the consumer chooses to use PIN-authentication, however, there is a second choice to be made. The average merchant location that accepts PIN debit accepts the cards of approximately three different PIN debit networks. ${ }^{6}$ And the average debit card can be run over 1.7 PIN debit networks. ${ }^{7}$ Hence, when a consumer presents his or her PIN debit card, there frequently is a choice of which PIN debit network to use. This choice is not made by the debit card holder. Instead, the choice is determined by contractual agreements between debit networks and their merchant and issuer members. ${ }^{8}$ The specification of which PIN network is used when there are multiple networks available is known as a routing rule.

There are several types of routing rule:

- Issuer Routing: The issuer selects the routing. A rational issuer will choose the network that charges the issuer the lowest transactions price, ceteris paribus. ${ }^{9}$

- Merchant Routing: A transaction routed according to merchant instructions.

\footnotetext{
${ }^{4}$ The broader issue of choosing among payment instruments (e.g., cash versus check versus debit card versus credit card) can also be viewed in these terms.

${ }^{5}$ EFT Data Book 2004, ATM 8 Debit News, September 11, 2003, at 3; The Nilson Report, No. 785, April 2003, at 6-7; Dove Consulting, Debit Issuer Survey: Cardholder Fees and Industry Outlook, August 2, 2002, at 16.

${ }^{6}$ Calculation based on data reported in EFT Data Book 2004, ATM \& Debit News, September 11, 2003; The Nilson Report, No. 785, April 2003; NYCE, "NYCE Statistics," available at www.nyce.net/newsroom_stats.html; Star, "About Star," available at www.star.com/?go=about.glance; and Visa, Visa Quarterly Report, August 2003, available at www.usa.visa.com/media/global/Q22003.pdf.

${ }^{7}$ Calculations based on data reported in EFT Data Book 2004, ATM \& Debit News, September 11, 2003.

${ }^{8}$ In the case of merchants, the formal contractual relationship with the network may actually be between the network and a financial institution (acquiring bank) that represents the merchant in dealings with the network.
}

${ }^{9}$ In practice, debit card issuers often face negative transactions prices. 
- Network Routing: Under this rule, a transaction is routed over a given network whenever feasible; that is, whenever both the card issuer and the merchant are affiliated with the network utilizing this routing rule.

Although not formally a routing rule, it is evident that if a merchant or issuer participates in only one debit network, then that exclusivity has the effect of choosing the routing. Hence, we will also explore the effects of a network's imposing the requirement that a merchant or card issuer or both belong uniquely to that network.

Below, we present our model using language appropriate to debit card networks. However, the issue also arises in other contexts. As noted above, for example, merchants often accept the credit cards of multiple networks. Although credit card issuers typically pick a single network for a given card to run over, a consumer may carry multiple credit cards in his or her wallet and choose which card to present when making payment at a merchant. Hence, credit cards networks can be thought of as having cardholder routing.

Similar issues arise in communications networks. For example, many people subscribe to both wireline and mobile telephone networks. The costs and benefits of message exchange incurred by the sender and receiver may depend on which network is used to complete a call. In this case, there is caller routing: through his or her choice of the number to dial, the caller determines whether the call is completed over a fixed-line or wireless network. The called party can influence the choice of routing both through his or her network subscription choices and by the choice of how widely to disseminate his or her different telephone numbers.

Routing issues also arise on the Internet because many local Internet service providers (ISPs) and web-hosting services are connected to multiple Internet backbone service providers. ${ }^{10}$ Shipping represents another example of routing choice; sometimes merchants choose the routing, sometimes consumers are given the choice, and the parties have some choice as to which networks they belong (e.g., in the Us, a consumer with a post office box has effectively chosen not to belong to the DHL or FedEx networks). The choice of language for an international conference and the choice of the word processing program in which to write a collaborative paper on routing rules are further examples in which two or more

\footnotetext{
${ }^{10}$ Crémer et al. (2000) provide an analysis of an ISP's incentives to connect to multiple Internet backbones.
} 
parties have to choose the common network over which to transact. Lastly, a subset of the routing regimes we consider are also relevant in settings such as game consoles and game software or operating systems and application software, a point we consider in greater depth in Section 6.1.

As the examples above illustrate, the choice of routing is determined by a combination of formal authority (i.e., which party is delegated the right to choose a network), network subscription decisions, and the distribution of information about available options. Our focus is on the interplay between formal authority and subscription decisions. We characterize users' equilibrium membership decisions for each of the routing rules identified above. Our central finding is that granting formal authority to one side of the market can increase the extent to which transactions run over the network preferred by the other side of the market. Intuitively, the side that does not have formal authority has stronger incentives to subscribe solely to its preferred network, which trumps the other side's authority.

We also characterize the networks' equilibrium choices of routing rules. Specifically, we establish conditions under which competing networks adopt routing rules that delegate the choice to one side of the market rather than imposing network routing or exclusivity. Intuitively, a delegated routing regime serves to get many people on both networks, which lessens the all-or-nothing type of competition that exists with network routing or exclusivity. Moreover, because many people join both networks, the volume of trade is greater.

Our analysis proceeds as follows. In the next section, we present a simple model of routing choice and network competition. We solve the model working backward in time, beginning with analysis of consumers network connection decisions in Section 3. We examine network price competition in Section 4. The equilibrium in the overall game, and the consequent choice of routing regime, are characterized in Section 5, which also examines the welfare properties of alternative equilibria. After considering four extensions of the model in Section 6, the paper closes with a brief concluding section. 


\section{A Model}

Two sides of a market need a network over which to complete a transaction. Let $A$ denote a member of the one side (e.g., a payment card user or a PIN debit card issuer) and $B$ denote a member of the other side (e.g., a merchant). Assume the two have been paired and that they wish to conduct a transaction with one another. There are two networks, denoted $X$ and $Y$, over which transactions can potentially be completed. $A$ and $B$ can actually complete a transaction over a given network only if they both belong to that network.

Let $a_{z}$ and $b_{z}$ denote the gross consumption benefits enjoyed by $A$ and $B$, respectively, if the transaction is completed over network $z, z \in\{X, Y\}$. If no transaction is completed, then the payoff to each side is zero.

The consumption benefits are randomly distributed. ${ }^{11}$ The realization of $\mathbf{a} \equiv\left(a_{X}, a_{Y}\right)$ is $A$ 's type, and the realization of $\mathbf{b} \equiv\left(b_{X}, b_{Y}\right)$ is $B$ 's type. We assume that $\mathbf{a} \sim F_{A}$ on the rectangle $\mathcal{I}_{A} \subset \mathbb{R}_{+}^{2}$ and $\mathbf{b} \sim F_{B}$ on the rectangle $\mathcal{I}_{B} \subset \mathbb{R}_{+}^{2} \cdot{ }^{12}$ Unless stated otherwise, we assume that the distribution functions are differentiable, with positive derivatives everywhere on the interior of their respective support rectangles. We also assume that $\mathbf{a}$ and $\mathbf{b}$ are distributed independently of each other; that is, knowledge of one side's type does not provide information about the other side's type.

The marginal costs of completing a transaction, $c$, are constant across all transactions and networks. We also assume that the networks incur no costs to add parties to their network.

It is taken to be common knowledge that the structure of the game is as follows:

1. The networks simultaneously choose their routing regimes (e.g., whether to have issuer, merchant, or network routing, or to have exclusive network membership).

2. After observing routing choices, the networks simultaneously choose their prices. In principle, these prices could include fixed membership (subscription) charges, as well

\footnotetext{
${ }^{11}$ In the debit context, different valuations can be interpreted as the extent to which merchants and issuers care about underlying differences in non-price network characteristics, such as consumer perceptions of the networks' brand names, network reliability and accuracy, and the provision of ancillary services.

${ }^{12}$ For expositional convenience we limit attention to non-negative values, but nothing in the analysis relies on this assumption.
} 
as per-transactions charges. In the present analysis, we restrict attention to pertransactions charges, denoted by $p_{z k}$, where $z \in\{X, Y\}$ and $k \in\{A, B\}$.

3. $A$ and $B$ learn the values of $\mathbf{a}$ and $\mathbf{b}$, respectively. These values are the private information of the individuals.

4. $A$ and $B$ simultaneously choose the networks to which to belong and, where they have the right, specify their routing choices (e.g., if both networks implement issuer routing, then the issuer specifies which network has priority).

5. $A$ and $B$ meet to conduct a transaction. If $A$ and $B$ are on different networks, then there is no transaction. If they have only one network in common, then the transaction is conducted on that network. If they have each joined both networks, then the network utilized is determined according to applicable routing rules. A member of a network cannot refuse a feasible trade that satisfies the routing rules. ${ }^{13}$

6. Payoffs are realized.

Our equilibrium concept is Bayesian Nash equilibrium refined by imposing a Trembling Hand Perfection requirement.

Our welfare measure is total surplus. Trade should occur if and only if

$$
\max _{z} a_{z}+b_{z} \geq c
$$

We refer to a situation in which trade fails to occur optimally as trade inefficiency. If trade is efficient, a transaction should be completed on network $Y$ rather than network $X$ if and only if

$$
a_{Y}+b_{Y} \geq a_{X}+b_{X}
$$

We will refer to a failure to abide by (1) as a routing inefficiency.

\footnotetext{
${ }^{13} \mathrm{We}$ assume that users cannot veto feasible trades in part to mirror actual practice in payment-card systems, where joining a network obligates a merchant to accept all transactions on that network. We also do it in part for expositional convenience: Relaxing the assumption would not change equilibrium payoffs or network utilization. It would, however, always have to be stated that users were not joining networks that offered them non-positive surplus or, equivalently, were joining them, but vetoing trade over them.
} 


\section{The Connection Continuation Game}

The surplus levels $A$ and $B$ derive if the transaction is completed on network $z$ are $s_{z A} \equiv$ $a_{z}-p_{z A}$ and $s_{z B} \equiv b_{z}-p_{z B}$, respectively. It proves convenient to define $h$ and $\ell$ for party $k$ as

$$
h=\underset{z \in\{X, Y\}}{\arg \max } s_{z k} \text { and } \ell=\underset{z \in\{X, Y\}}{\arg \min } s_{z k} .
$$

When $s_{h k}>0$, we can define the ratio of the surplus on the less-preferred network to the more-preferred network for party $k$ :

$$
r_{k}=\frac{s_{\ell k}}{s_{h k}} .
$$

In what follows, it should be understood that the prices have been set so that there is a positive probability that each of $A$ and $B$ can gain surplus from transacting over either network. That is, $\operatorname{Pr}\left\{s_{z k}>0\right\}>0$ for all $(z, k)$ pairs.

There is never any gain to $k$ from joining network $z$ if $s_{z k} \leq 0$. Moreover, joining a network for which $s_{z k} \leq 0$ could be distinctly disadvantageous (e.g., when $s_{X k}>0 \geq s_{Y k}$ but the operative routing rule requires that, if the parties are on both networks, trade be on $Y$ ). Given the possibility of trembles by the other party, party $k$ does better not to join network $z$ if $s_{z k}<0$ because $k$ can never be assured trade will not go over $z$. Because even trade that generates positive surplus with small probability beats no trade with certainty, the possibility of trembles means that a strategy of joining neither network is strictly dominated if a party would derive positive surplus from completing a transaction on one or both networks.

We have established:

Lemma 1 For $k=A, B$ :

(i) If $s_{h k}<0$, then party $k$ joins neither network.

(ii) If $s_{h k}>0>s_{\ell k}$, then party $k$ must join network $h$ and not join $\ell$.

(iii) If $s_{\ell k}>0$, party $k$ must join at least one of the two networks.

As we will see, whether $k$ joins $X, Y$, or both in (iii) depends on the nature of the routing rules, among other factors. In what follows, we assume that user $k$ stays off network $z$ when 
$s_{z k}=0$ (even if $k$ controls the routing choice) because $k$ can never benefit from joining $z$. In any case, $s_{z k}=0$ is a measure-zero event and its treatment is, therefore, immaterial.

\subsection{One-Side-Chooses Routing}

Some routing regimes, such as issuer or merchant routing in the PIN-debit industry, grant one side of the transaction the right to select the network utilized when both $A$ and $B$ belong to both networks. If both networks choose an issuer-routing regime or both choose a merchantrouting regime, then the interpretation of contracts between networks and their members is obvious. However, if one network chooses merchant routing and the other chooses issuer routing, then contractual conflicts can arise. Suppose, for instance, that issuer $A$ chooses network $X$ under $X$ 's issuer-routing regime, but merchant $B$ gives priority to network $Y$ under $Y$ 's merchant-routing regime. ${ }^{14}$ There is no obvious view as to how such conflicts should be resolved. In this section, we therefore limit attention to the situation in which both networks have chosen the same routing regime.

Without loss of generality, we consider only the case in which $A$ is given the routing choice by both networks. ${ }^{15}$ Clearly, when given the choice, $A$ selects $X$ if $s_{X A}>s_{Y A}$ and $Y$ if $s_{X A}<s_{Y A} \cdot{ }^{16}$ Moreover, given that $A$ makes the choice when multiple networks are available, and given that $B$ may tremble, the only rational strategy for $A$ is to join any network for which $s_{z A}>0$. Figure 1 illustrates $A$ 's network membership and routing choices as a function of its type. Observe that $A$ 's optimal strategy is independent of $B$ 's strategy.

The more subtle question is: what network choice does $B$ make when both $s_{X B}$ and $s_{Y B}$ are positive? To answer this question, consider the expected value of different network membership choices, making use of $A$ 's equilibrium strategy and the distribution of a. Let $\alpha_{X}$ denote the resulting probability that $A$ joins network $X$ only, $\alpha_{Y}$ the probability that $A$ joins network $Y$ only, and $\alpha_{X Y}$ the probability that $A$ joins both networks. $B$ 's respective

\footnotetext{
${ }^{14}$ For the credit-card industry, $A$ can be interpreted as the card holder.

${ }^{15}$ The analysis when $B$ is given the choice is symmetric.

${ }^{16}$ Because the event $s_{X k}=s_{Y k}$ is a measure-zero event, it is immaterial how we resolve indifference.
} 


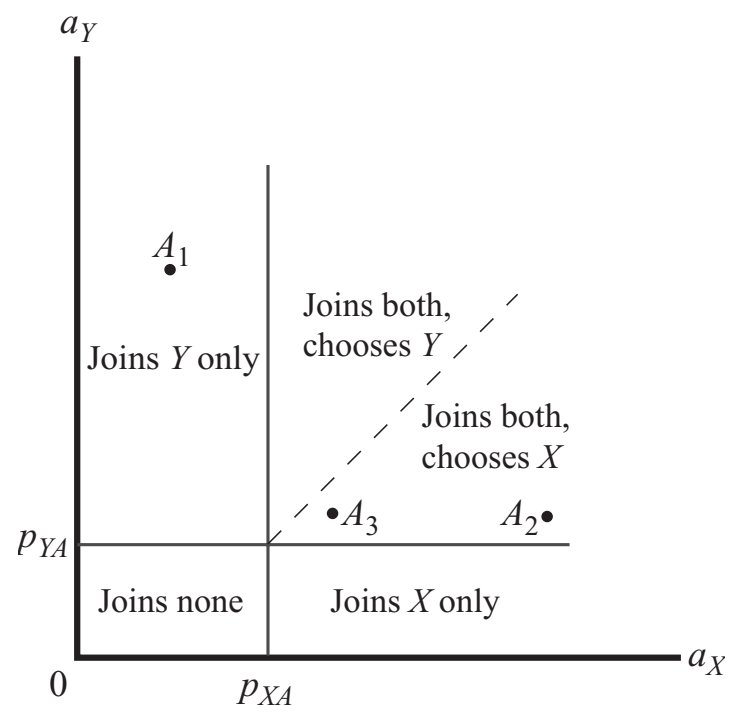

Figure 1: $A$ 's equilibrium strategy as a function of type under an $A$-chooses routing regime. The dotted ray has slope 1 .

expected payoffs from joining $X$ only, $Y$ only, and both networks are

$$
\begin{aligned}
& \pi_{X}^{B}=s_{X B}\left(\alpha_{X}+\alpha_{X Y}\right), \\
& \pi_{Y}^{B}=s_{Y B}\left(\alpha_{Y}+\alpha_{X Y}\right),
\end{aligned}
$$

and

$$
\pi_{X Y}^{B}=s_{X B} \alpha_{X}+\alpha_{X Y}\left(\lambda_{X} s_{X B}+\lambda_{Y} s_{Y B}\right)+s_{Y B} \alpha_{Y}
$$

where $\lambda_{z}$ is the probability that $A$ prefers network $z$ conditional on $A$ 's having chosen to join both networks. By definition, $\lambda_{X}+\lambda_{Y} \equiv 1$.

It is apparent that $\pi_{\ell}^{B} \leq \pi_{X Y}^{B}$. Hence the relevant comparison for $B$ is between $\pi_{h}^{B}$ and $\pi_{X Y}^{B}$. He therefore joins both networks if and only if

$$
s_{h B}\left(\alpha_{h}+\alpha_{X Y}\right) \leq s_{h B} \alpha_{h}+\alpha_{X Y}\left(\lambda_{h} s_{h B}+\lambda_{\ell} s_{\ell B}\right)+s_{\ell B} \alpha_{\ell},
$$

which, using $s_{\ell B}=r_{B} s_{h B}$ and $\lambda_{h}=1-\lambda_{\ell}$, is equivalent to

$$
0 \leq \alpha_{X Y} \lambda_{\ell}\left(r_{B}-1\right)+r_{B} \alpha_{\ell}
$$




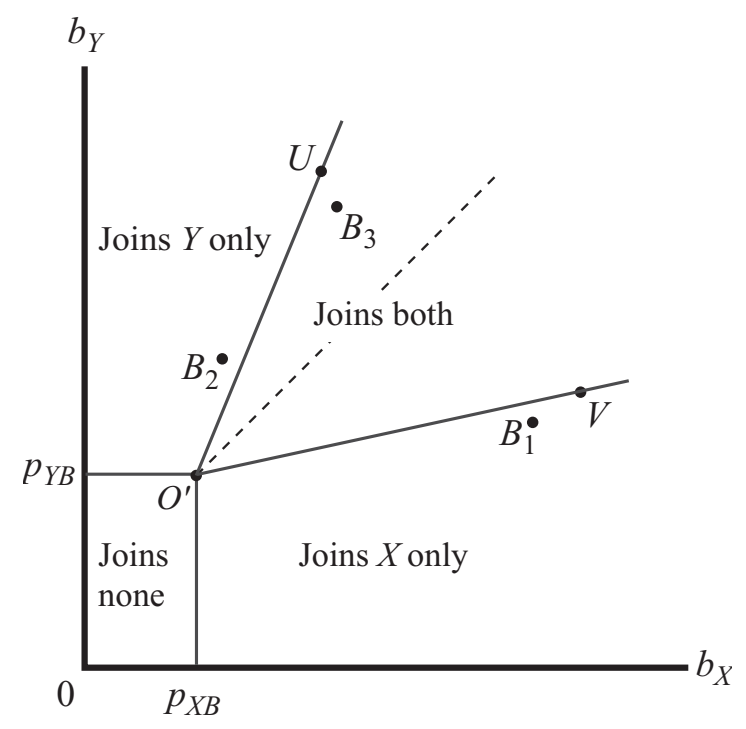

Figure 2: $B$ 's equilibrium strategy as a function of type under an $A$-chooses routing regime. The dotted ray has slope 1 .

From this last expression, we see that $B$ should join both networks when $B$ is largely indifferent between them (i.e., $r_{B}$ close to one) or it is relatively likely that $A$ will be on $B$ 's less-preferred network only (i.e., $\alpha_{\ell}$ large). Otherwise, $B$ should join only its preferred network to avoid the risk of having to transact on its less-preferred network. Figure 2 illustrates $B$ 's strategy as a function of its type.

We have established:

Proposition 1 When A controls the routing choice, the unique equilibrium is:

(i) If $s_{h k} \leq 0, k$ joins neither network, $k=A, B$;

(ii) If $s_{h k}>0 \geq s_{\ell k}, k$ joins network $h$ only, $k=A, B$;

(iii) If $s_{\ell A}>0$, then $A$ joins both networks.

(iv) If $s_{\ell B}>0$ and

$$
0 \leq \alpha_{X Y} \lambda_{\ell}\left(r_{B}-1\right)+r_{B} \alpha_{\ell}
$$

then $B$ joins both networks; and 
(v) If $s_{\ell B}>0$ and expression (2) does not hold, then $B$ joins only network $h$.

Figures $1 \& 2$ illustrate this equilibrium. In Figure 2, the slopes of the rays $\overrightarrow{O^{\prime} U}$ and $\overrightarrow{O^{\prime} V}$ are

$$
\frac{\alpha_{X Y} \lambda_{X}+\alpha_{X}}{\alpha_{X Y} \lambda_{X}} \text { and } \frac{\alpha_{X Y} \lambda_{Y}}{\alpha_{X Y} \lambda_{Y}+\alpha_{Y}},
$$

respectively (where the slopes derive from expression (2) above).

Consider the consequence for $B$ 's strategy of changing one of the prices $A$ faces. If $p_{X} A$ falls, then, from Figure 1, $\alpha_{X}$ and $\alpha_{X Y} \lambda_{X}$ increase, $\alpha_{Y}$ decreases, and the effect on $\alpha_{X Y} \lambda_{Y}$ is ambiguous. In addition, $\alpha_{X Y} \lambda_{Y}+\alpha_{Y}$ decreases and $\alpha_{X Y} \lambda_{X}+\alpha_{X}$ increases. Consequently, from expression (3), the implications for $B$ 's strategies are a priori indeterminant. Consider $B$ 's margin between joining $X$ only or joining both. Because $\alpha_{Y}$ is smaller, $B$ 's risk of no trade if it were to join solely its preferred network, $X$, has gone down, which pushes it towards joining $X$ only. On the other hand, if $\alpha_{X Y} \lambda_{Y}$ has gone down, then $B$ 's risk, when it has joined both networks, of being paired with an $A$ that wishes to trade on $B$ 's less-preferred network has gone down. This would then push $B$ toward joining both networks. Similar opposing factors apply for a $B$ on the margin between joining $Y$ only or joining both.

A sense for the nature of this equilibrium can be gained by considering the limiting case in which $p_{X A}=p_{Y A}=0$. In this case, all types of $A$ join both networks, so $\alpha_{X}=\alpha_{Y}=0$; hence, expression (2) becomes $r_{B} \geq 1$, which, at most, holds for a set of $\mathbf{b}$ of measure zero. In terms of Figure 2, we can see from expression (3) that the rays $\overrightarrow{O^{\prime} U}$ and $\overrightarrow{O^{\prime} T}$ collapse to the dotted line. $B$ therefore joins its preferred network only. In this limiting case, we see that although $A$ has the nominal choice of routing, $B$ actually has the real choice of routing: in equilibrium, $A$ and $B$ conduct their transaction on $B$ 's preferred network. In essence, $A$ and $B$ play a game of chicken in which only $A$ 's car has a steering wheel; not surprisingly, $A$ is thus the "loser."

Expressed another way, network membership decisions can trump formal authority over routing decisions. A user who cannot control the routing choice has greater incentives to join solely its preferred network than does a user who can choose among networks on a transaction-by-transaction basis. The consequences of this fact can be seen by considering a notional cheap-talk game. Suppose that each side of the market can make a speech to the 
other side before making its network membership decisions. When $A$ has the formal authority over routing, $A$ cannot credibly threaten to join solely its preferred network because joining the other network creates an option that $A$ has the discretion to exercise. But $B$ can announce that it will join solely its preferred network because it knows that $A$ will join both networks and thus the option value to $B$ of joining both is limited. Moreover, if $B$ does create the option by joining both networks, $A$ may exercise it in a way unfavorable to $B$.

Figures 1 and 2 also illustrate some of the welfare properties of this equilibrium. Let $A_{n}$ and $B_{n}$ denote specific types of $A$ and $B$. Assume that $p_{z A}+p_{z B}=c$ for both $z \cdot{ }^{17}$ If $A_{1}$ is paired with $B_{1}$, then a trade inefficiency occurs; $a_{Y}+b_{Y}>c$, yet no transaction is completed. If $A_{2}$ is paired with $B_{2}$, then a routing inefficiency occurs; as drawn, $a_{X}+b_{X}>a_{Y}+b_{Y}$, yet the transaction is conducted on $Y$ ( $B_{2}$ 's preferred network). This distortion arises because $B$ ignores the adverse effect on $A$ from $B$ 's decision to force trade over $Y$ by joining that network solely. Routing inefficiency also occurs when $A_{3}$ and $B_{3}$ are paired; $a_{Y}+b_{Y}>a_{X}+b_{X}$, yet the transaction is conducted on $X$ ( $A_{3}$ 's preferred network). Here, $A$ ignores $B$ 's preferences when $A$ picks the routing.

\subsection{Network Routing}

An alternative regime for network selection when $A$ and $B$ have joined both networks is network routing, under which a network stipulates that, whenever possible, its members must conduct their transactions on it.

If both networks specify network routing, then conflict arises if $A$ and $B$ both subscribe to both networks. Because such conflict would lead to a breach of contract with at least one of the networks, we assume each party signs with at most one network when both networks impose network routing. ${ }^{18}$ Observe, however, that if only one network adopts

${ }^{17}$ As drawn, the figures illustrate the case in which

$$
p_{X A}+p_{X B}=p_{Y A}+p_{Y B}
$$

\footnotetext{
${ }^{18}$ If the parties could somehow coordinate, it would be possible for only one side (say $A$ ) to sign with at most one network, while the other side $(B)$ could then sign with both with no risk of breach of contract. That case is, however, identical to a regime in which $A$ is limited to exclusive membership in at most one network, which we consider below in Section 3.3.
} 
network routing, while the other adopts another routing regime (e.g., merchant routing), then it is possible for a party to join both networks.

\subsubsection{Both networks adopt network routing}

Suppose both networks have adopted network routing. Then each user can join at most one network. The following result indicates that this exclusivity can sometimes lead to tipping.

Lemma 2 Suppose that both networks implement network routing.

(i) If and only if $\operatorname{Pr}\left\{s_{X k} \leq 0\right.$ and $\left.s_{Y k}>0\right\}=0$ for $k=A, B$, then there exists an equilibrium of the connection continuation game in which any user who joins a network joins only network $X$.

(ii) If and only if $\operatorname{Pr}\left\{s_{Y k} \leq 0\right.$ and $\left.s_{X k}>0\right\}=0$ for $k=A, B$, then there exists an equilibrium of the connection continuation game in which any user who joins a network joins only network $Y$.

The intuition underlying this result is straightforward. Consider the $X$-only equilibrium. If the condition stated in the lemma did not hold, a positive measure of types on one side of the market would have incentives to join network $Y$ in hopes of trembles by the other side of the market. When the condition is satisfied, there is no reason for anyone to join network $Y$ given that there is a vanishingly small probability that anyone from the other side of the market will be on that network as a potential trading partner. A parallel analysis applies to the $Y$-only equilibrium.

We next consider the possibility of equilibria in which both networks obtain members. Specifically, we now assume that

$$
\operatorname{Pr}\left\{s_{X k} \leq 0 \text { and } s_{Y k}>0\right\}>0 \text { and } \operatorname{Pr}\left\{s_{Y k} \leq 0 \text { and } s_{X k}>0\right\}>0 \text { for } k=A, B .
$$

This assumption ensures that there are types of $A$ and $B$ that are realized with positive probability that choose to be on only network $X$ and user types that are realized with positive probability that choose to be on only network $Y$.

Consider a user for whom $s_{h k}>0$. This user's best response is: 


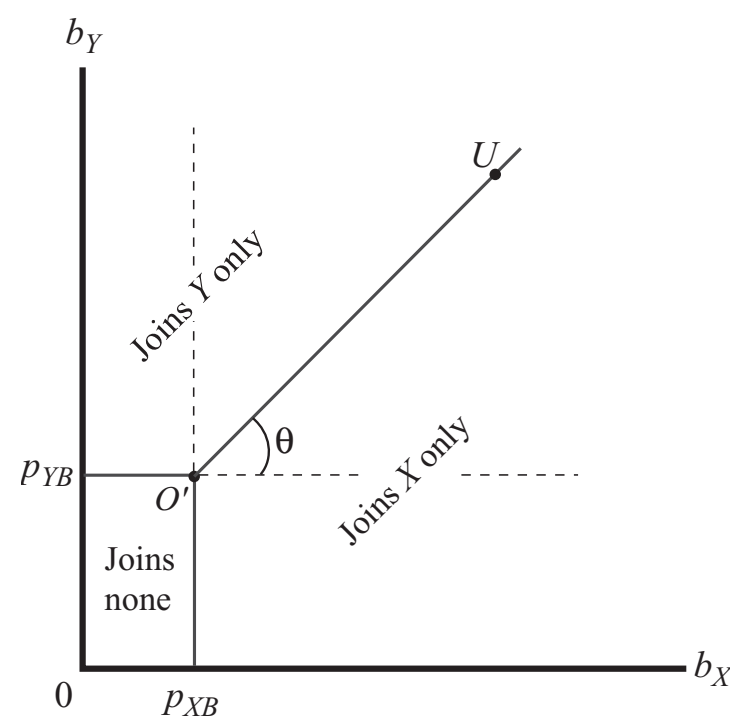

Figure 3: $B$ 's equilibrium strategy as a function of type when both networks adopt network routing.

For $A$ :

Join $X$ if $\beta_{X} s_{X A}>\beta_{Y} s_{Y A}$

Join $Y$ if $\beta_{X} s_{X A} \leq \beta_{Y} s_{Y A}$.

For $B$ :

Join $X$ if $\alpha_{X} s_{X B}>\alpha_{Y} s_{Y B}$

Join $Y$ if $\alpha_{X} s_{X B} \leq \alpha_{Y} s_{Y B}$.

Recall, $\alpha_{z}$ is the equilibrium probability that $A$ joins $z$ only, and $\beta_{z}$ is the equilibrium probability that $B$ joins $z$ only. From assumption (4) and Lemma $1, \alpha_{z}>0$ and $\beta_{z}>0$ for both $z$. Figure 3 illustrates for party $B$ (the picture for $A$ is similar).

Observe from Figure 3 that we can conceive of B's best response as being the placement of the ray $\overrightarrow{O^{\prime} U}$ that divides the join- $X$-only region from the join- $Y$-only region. Because that ray is always "hinged" at $O^{\prime}$, placing the segment is equivalent to choosing the angle $\theta \in\left[0^{\circ}, 90^{\circ}\right]$.

Let $\Theta_{k}(\cdot)$ be party $k$ 's best response function. Consider $\Theta_{A}(\cdot)$. B's strategy, $\theta_{B}$, defines the regions join- $X$-only and join- $Y$-only and, thus, the probabilities $\beta_{X}$ and $\beta_{Y}$. From above,

$$
\Theta_{A}\left(\theta_{B}\right)=\arctan \left(\frac{\beta_{X}\left(\theta_{B}\right)}{\beta_{Y}\left(\theta_{B}\right)}\right) \text {. }
$$




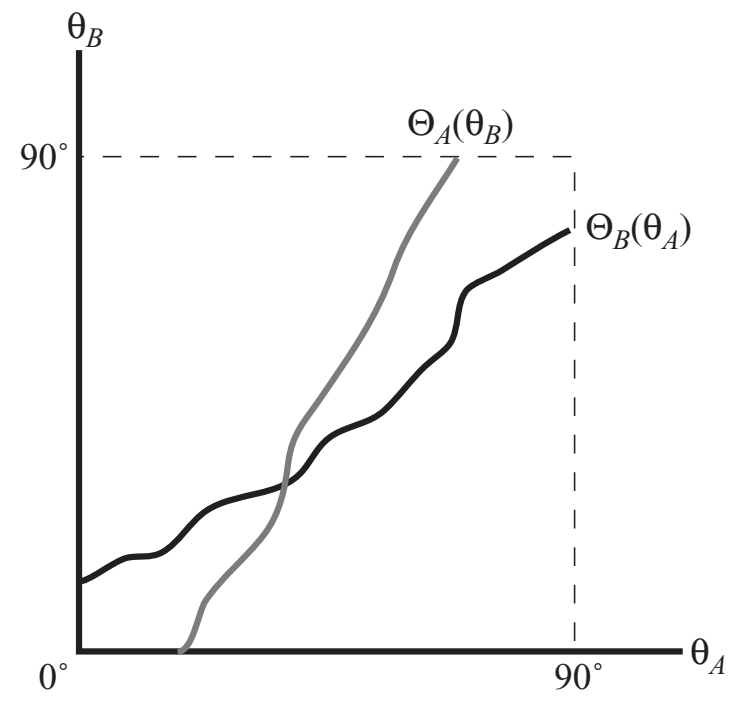

Figure 4: Best-response functions in $\theta$ when both networks stipulate network routing.

Because the underlying distribution is continuous, $\beta_{X}(\cdot)$ and $\beta_{Y}(\cdot)$ are continuous, as is their ratio. It follows that $\Theta_{A}(\cdot)$ is continuous. It is also readily shown to be increasing. By assumption (4) and Lemma $1, \beta_{X}(\cdot)$ and $\beta_{Y}(\cdot)$ are bounded away from zero for all $\theta_{B} \in\left[0^{\circ}, 90^{\circ}\right]$. Hence,

$$
\Theta_{A}\left(0^{\circ}\right)>0^{\circ} \text { and } \Theta_{A}\left(90^{\circ}\right)<90^{\circ} \text {. }
$$

Using expression (6) and the corresponding analysis for $B$, we know the best-response curves will resemble those shown in Figure 4, at least with respect to their end points. These end points and the functions' continuity guarantee that the functions cross; hence, an equilibrium exists.

Proposition 2 Suppose both networks implement network routing and that assumption (4) holds. Then there is an equilibrium of the connection continuation game in which:

(i) If $s_{h k} \leq 0$, then $k$ joins neither network, $k=A, B$;

(ii) If $s_{h k}>0$, then $k$ joins only network $X$ if $s_{Y k}<s_{X k} \tan \left(\theta_{k}\right)$, and $k$ joins only network $Y$ if $s_{Y k} \geq s_{X k} \tan \left(\theta_{k}\right), k=A, B$; and 
(iii) The angles $\theta_{A}$ and $\theta_{B}$ lie in $\left(0^{\circ}, 90^{\circ}\right)$ and satisfy the relations

$$
\tan \left(\theta_{A}\right)=\frac{\beta_{X}\left(\theta_{B}\right)}{\beta_{Y}\left(\theta_{B}\right)} \text { and } \tan \left(\theta_{B}\right)=\frac{\alpha_{X}\left(\theta_{A}\right)}{\alpha_{Y}\left(\theta_{A}\right)},
$$

where $\alpha_{z}\left(\theta_{A}\right)$ is the probability of realizing types of $A$ who join network $z$ under condition (ii); and $\beta_{z}\left(\theta_{B}\right)$ is the probability of realizing types of $B$ who join network $z$ under condition (ii).

Observe that the use of network routing can lead to a large amount of trade inefficiency. Because the two sides of the market have heterogeneous preferences and are forced to make exclusive choices, there can be many instances in which trade would be mutually beneficial, but the two sides have chosen to be on different networks and thus cannot transact.

Routing inefficiency is also possible unless all three of the following conditions are satisfied: (a) $\theta_{A}=\theta_{B}=45^{\circ}$; (b) $p_{X A}=p_{Y A}$; and (c) $p_{X B}=p_{Y B}$. Without conditions (b) and (c), the parties' choices of network will reflect the relative transactions prices of the two networks, rather than solely the gross consumption benefits. Condition (a) is needed to insure that neither $A$ nor $B$ is on its less preferred network. If one were, then routing inefficiency could readily arise. This possibility can be seen by considering types near the $45^{\circ}$ line in Figure 3.

\subsubsection{Only one network stipulates network routing}

Now, suppose that only one network, say $X$, stipulates network routing. Whether $Y$ selects an $A$-chooses regime or a $B$-chooses regime is irrelevant because, whenever the transaction can go over both networks, the party with the choice must choose $X$ to avoid breaching its contract with that network. ${ }^{19}$

In this regime, there cannot be equilibria in which only one network attracts members: If party $k$ thought there were even a minuscule probability the other party would be on the other network (as well or only), then a measure of types of party $k$ bounded away from zero would wish to be on both networks, as the ensuing analysis will confirm. Hence, a one-

\footnotetext{
${ }^{19}$ If network $Y$ requires exclusivity from one side, then, as we show below, it would be as if both networks had required exclusivity from that side only. See Section 3.3.
} 
network-only-has-members equilibrium cannot be the limit of the equilibria as the trembles go to zero.

We now construct a trembling-perfect Nash equilibrium. In doing so, we begin with the supposition, which we will confirm holds in equilibrium, that

$$
\alpha_{Y}>0, \alpha_{X Y}>0, \beta_{Y}>0, \text { and } \beta_{X Y}>0
$$

where the $\alpha_{z}$ and $\beta_{z}$ s are as defined above; and where $\alpha_{X Y}$ and $\beta_{X Y}$ are the equilibrium probabilities that $A$ joins both and $B$ joins both, respectively.

As before, party $k$ joins neither network if $s_{h k} \leq 0$ and joins only network $h$ if $s_{h k}>0 \geq$ $s_{\ell k}$. The only case remaining, therefore, is $s_{\ell k}>0$. Consider $A$ 's decision. ${ }^{20}$ When $s_{\ell A}>0$, the payoffs for $A$ are

$$
\begin{aligned}
\pi_{X}^{A}=s_{X A}\left(\beta_{X}+\beta_{X Y}\right) & (X \text { only }), \\
\pi_{Y}^{A}=s_{Y A}\left(\beta_{Y}+\beta_{X Y}\right) & (Y \text { only }), \text { and } \\
\pi_{X Y}^{A}=s_{X A}\left(\beta_{X}+\beta_{X Y}\right)+s_{Y A} \beta_{Y} & \text { (both) }
\end{aligned}
$$

If $s_{X A} \geq s_{Y A}$, then, in the light of (7), A's unique best response is to join both networks. Intuitively, $A$ joins not only its preferred network, $X$, but also $Y$, because a transaction will go over $Y$ only if $B$ is not on $X$. If $s_{X A}<s_{Y A}$, then joining network $X$ only is strictly dominated. Observe that we have established that $A$ joins solely network $X$ if and only if $s_{X A}>0 \geq s_{Y A}$. The question remaining is under what conditions should $A$ join $Y$ only or join both when $s_{X A}<s_{Y A}$ ? That decision depends on whether

$$
\begin{aligned}
0 & \gtrless \pi_{X Y}^{A}-\pi_{Y}^{A} \\
& =s_{X A} \beta_{X}-\beta_{X Y}\left(s_{Y A}-s_{X A}\right),
\end{aligned}
$$

which is equivalent to

$$
s_{Y A} \gtrless s_{X A} \frac{\beta_{X}+\beta_{X Y}}{\beta_{X Y}} .
$$

\footnotetext{
${ }^{20}$ The analysis for $B$ is symmetric.
} 


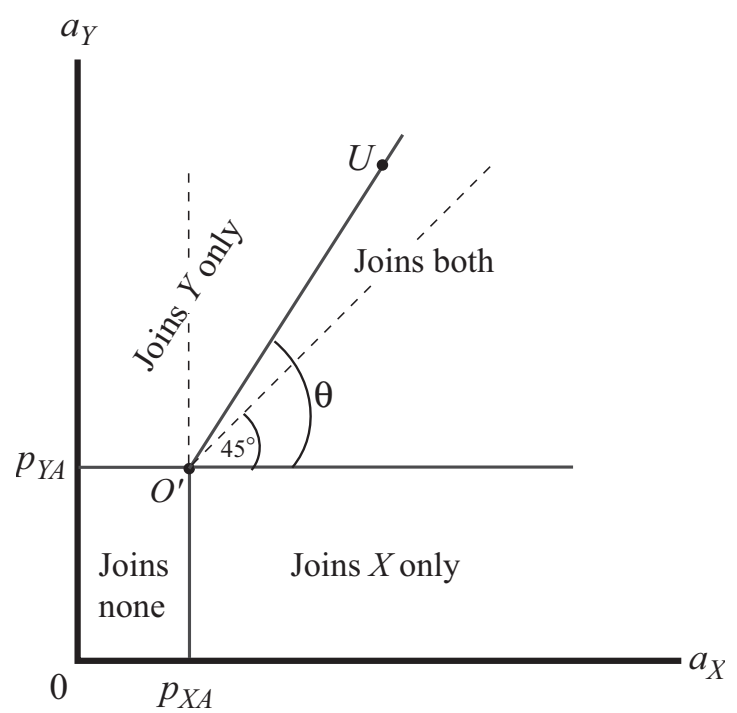

Figure 5: $A$ 's equilibrium strategy as a function of type when only network $X$ stipulates network routing.

Figure 5 illustrates. The fraction in expression (8) is the slope of the ray $\overrightarrow{O^{\prime} U}$ that divides the join- $Y$-only region from the join-both region. The angle $\theta$ is, thus, the arctan of that fraction. Observe that $\theta$ is greater than forty-five degrees for any $\beta_{X}>0$. Types in the region between the forty-five degree ray and the $\theta$ ray would prefer that the transaction take place over network $Y$, and joining $X$ risks having the transaction routed over that network even though $A$ and $B$ both are on $Y$ as well. However, the benefit to these $A$-types of joining network $X$ is that it allows them to transact with $B$-types that join only $X$.

Let $\Omega_{k}(\cdot)$ be party $k$ 's best-response function. Consider $\Omega_{A}(\cdot)$ (the analysis for $B$ is symmetric). $B$ 's strategy $\theta_{B}$ defines the join-both region and, thus, the probability $\beta_{X Y}$. From (8),

$$
\Omega_{A}\left(\theta_{B}\right)=\arctan \left(\frac{\beta_{X}+\beta_{X Y}\left(\theta_{B}\right)}{\beta_{X Y}\left(\theta_{B}\right)}\right) .
$$

Because the underlying distributions are continuous, $\beta_{X Y}\left(\theta_{B}\right)$ is continuous, and, thus, so too is the argument of arctan in (9). Hence, $\Omega_{A}(\cdot)$ is continuous. It is readily shown to be decreasing (unlike $\Theta_{A}(\cdot)$ or $\Theta_{B}(\cdot)$ ). As the above analysis demonstrated (see also Figure 5), 


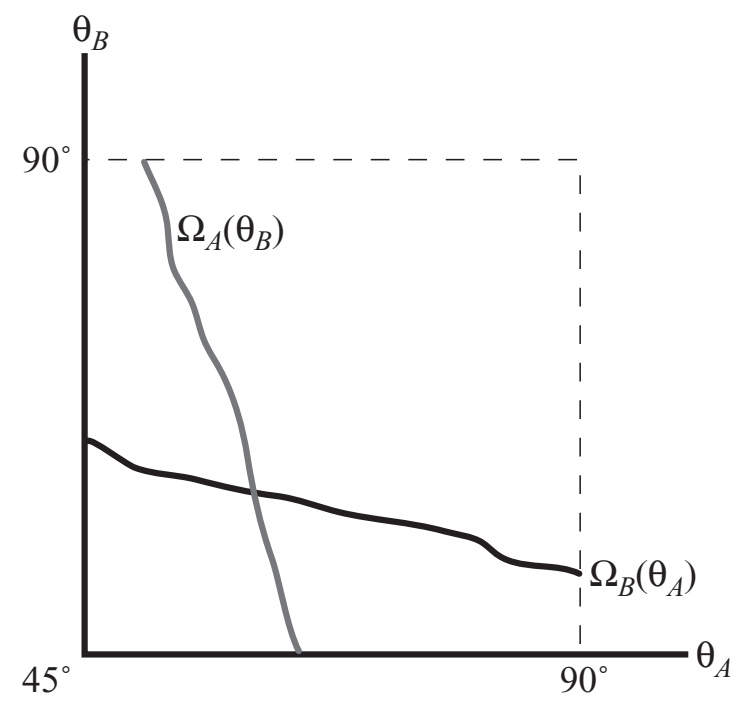

Figure 6: Best-response functions in $\theta$ when $X$ only stipulates network routing.

$\theta_{B} \in\left[45^{\circ}, 90^{\circ}\right]$. Because $\beta_{X Y}>0$,

$$
\Omega_{A}\left(\left[45^{\circ}, 90^{\circ}\right]\right) \subset\left[45^{\circ}, 90^{\circ}\right)
$$

Hence, $\Omega_{A}(\cdot)$ and $\Omega_{B}(\cdot)$ will resemble those shown in Figure 6 - at least with respect to their end points. That and their continuity guarantees that they cross; hence, an equilibrium exists.

Proposition 3 Suppose that network $X$ stipulates network routing and network $Y$ stipulates either $A$ or $B$ routing. Then, for $k=A, B$, there exists an equilibrium of the connection continuation game in which:

(i) If $s_{h k} \leq 0$, then $k$ joins neither network;

(ii) If $s_{h k}>0 \geq s_{\ell k}$, then $k$ joins network $h$ only;

(iii) If $s_{X k}>s_{Y k}>0$, then $k$ joins both networks;

(iv) If $s_{Y k}>s_{X k} \tan \left(\theta_{k}\right)>0$, then $k$ joins $Y$ only; and

(v) If $s_{X k} \tan \left(\theta_{k}\right) \geq s_{Y k}>s_{X k}>0$, then $k$ joins both networks. 
The angles $\theta_{A}$ and $\theta_{B}$ are in $\left[45^{\circ}, 90^{\circ}\right)$ and satisfy the relations

$$
\tan \left(\theta_{A}\right)=\frac{\beta_{X}+\beta_{X Y}\left(\theta_{B}\right)}{\beta_{X Y}\left(\theta_{B}\right)} \text { and } \tan \left(\theta_{B}\right)=\frac{\alpha_{X}+\alpha_{X Y}\left(\theta_{A}\right)}{\alpha_{X Y}\left(\theta_{A}\right)}
$$

where $\alpha_{X Y}\left(\theta_{A}\right)$ is the probability of realizing types of $A$ who join both networks under conditions (iii) and $(v)$; and $\beta_{X Y}\left(\theta_{B}\right)$ is the probability of realizing types of $B$ who join both networks under conditions (iii) and (v).

It is readily seen from Figure 5 that both trade and routing inefficiency can arise in equilibrium.

There is an intuition among some debit industry participants that network routing leads to a network's getting a disproportionate share of the transactions because any transaction that could run over that network must run over that network. As we will now show, this intuition is seriously incomplete and, thus, misleading.

Consider the market shares of $X$ and $Y$ when they are otherwise in identical positions. That is, when they charge equal prices (i.e., $p_{X A}=p_{Y A} \equiv p_{A}$ and $p_{X B}=p_{Y B} \equiv p_{B}$ ) and neither is inherently preferred in expectation. A general analysis is intractable, but we can determine shares for specific distributions of user types.

First, suppose that there is no intrinsic network differentiation and all of the mass for both $A$ and $B$ lies on their respective forty-five degree lines. Then any given user either joins both networks or joins neither. Thus, if network $X$ alone implements a network routing rule, all of the transactions take place on that network, as suggested by the intuitive argument above.

Suppose, however, that the two sides' types are uniformly distributed on a unit square. In this case, it can be shown that

$$
\begin{aligned}
& \operatorname{Pr}\{\text { trade occurs on } Y\}-\operatorname{Pr}\{\text { trade occurs on } X\} \\
& \quad=\frac{\left(1-p_{A}\right)\left(1-p_{B}\right)\left(1-p_{A} p_{B}\right)\left(Q-\sqrt{Q^{2}-8\left(1-p_{A}^{2}\right)\left(1-p_{B}^{2}\right)}\right)}{4\left(1+p_{A}\right)\left(1+p_{B}\right)},
\end{aligned}
$$

where

$$
Q \equiv 3+p_{A}+p_{B}-p_{A} p_{B}
$$


It is clear that expression (10) is positive, which means that $Y$ 's expected share of transactions exceeds $X$ 's; by being the only network to stipulate network routing, $X$ does worse than $Y$ when the networks are otherwise identical.

Corollary 1 Suppose that network $X$ stipulates network routing, but network $Y$ does not. If $X$ and $Y$ charge the same prices, and the distributions of user types are uniform on the unit square, then the equilibrium expected probability of trade is higher on network $Y$ than on network $X$.

This result is a further illustration of the fact that network membership decisions can override formal authority. Specifically, a user who prefers $X$ but would get positive surplus from a transaction on $Y$ (i.e., $s_{X k}>s_{Y k}>0$ ) should join both networks because network $Y$ will be used only as a last resort. However, a user that prefers $Y$ may refuse to join $X$ because joining could result in routing over $X$ even when both users are members of $Y$. Thus, more users join only network $Y$ than join only network $X$.

\subsection{One-Sided Exclusivity}

As already discussed, either $A$ or $B$ may choose to join only one of the two networks, and this exclusivity becomes a de facto routing rule. The networks could also require exclusivity as a condition of joining a network. Were one or both networks to require exclusivity of both sides of the market, then the resulting continuation game would be identical to the game in which both networks stipulate network routing; a game analyzed above. Hence, in this subsection, we restrict attention to continuation games in which one or both of the networks requires exclusive relationships with only one side of the market. In the debit card industry, for example, merchants typically accept cards issued on many networks, but card issuers are sometimes given incentives to issue cards on only one debit network.

There are three cases to consider:

1. Both networks require exclusivity of the same side of the market (e.g., issuers).

2. One network requires exclusivity of $A$ and the other requires exclusivity of $B$. 


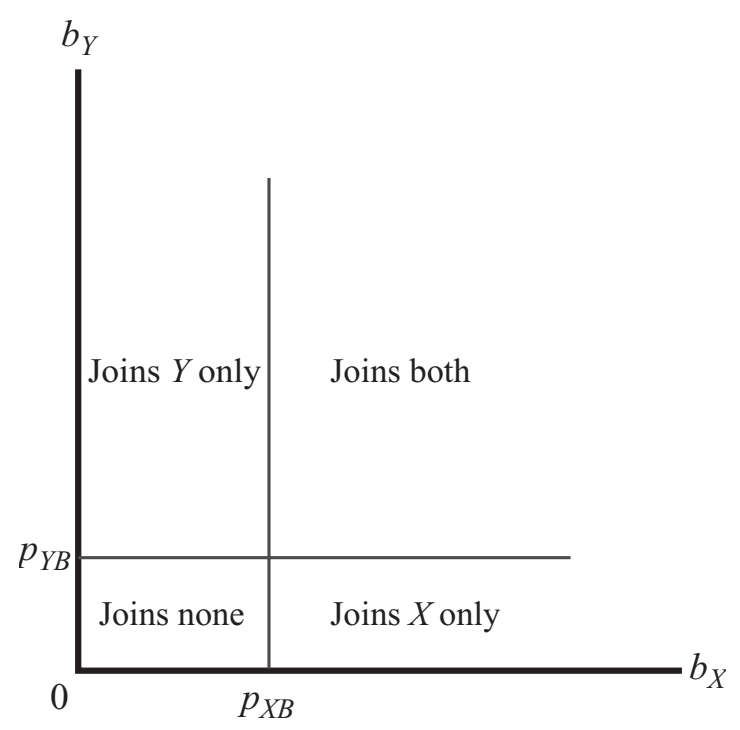

Figure 7: $B$ 's equilibrium strategy as a function of type when both networks require exclusivity of $A$.

3. One network requires exclusivity of one side and the other network adopts one of the alternative routing rules.

In case 2 , each side must choose one or the other network. Hence, case 2 is equivalent to the continuation game in which both networks stipulate network routing, which was analyzed above.

In case 3, one side cannot belong to both networks. Hence, it is irrelevant what routing regime the network that doesn't require exclusivity chooses (call that network $z$ ). Moreover, because the one side can't be on both networks, it is as if network $z$ requires exclusivity of that side. Therefore, cases 1 and 3 yield the same continuation game.

Consider case 1 . Without loss of generality, suppose that both networks require $A$ to make an exclusive choice of network. Given the prospect of trembles by $A, B$ 's strategy must be to join any network for which $s_{z B}>0$ and not to join any network for which $s_{z B}<0$. Observe that this strategy is optimal regardless of $A$ 's strategy. Figure 7 illustrates.

Given $B$ 's strategy, $A$ chooses the network that maximizes its expected surplus using the probabilities induced by $B$ 's strategy and distribution of types. That is, $A$ compares $\left(\beta_{X}+\beta_{X Y}\right) s_{X A}$ with $\left(\beta_{Y}+\beta_{X Y}\right) s_{Y A}$. Figure 8 illustrates. Observe the slope of the ray $\overrightarrow{O^{\prime} U}$ 


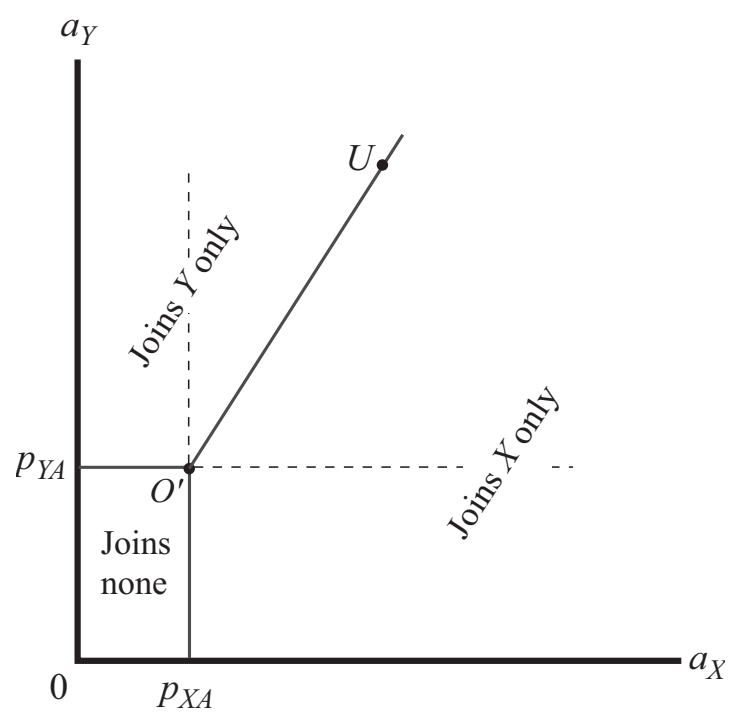

Figure 8: $A$ 's equilibrium strategy as a function of type when both networks require exclusivity of $A$. The slope of ray $\overrightarrow{O^{\prime} U}$ is given by expression (11).

is given by

$$
\frac{\beta_{X}+\beta_{X Y}}{\beta_{Y}+\beta_{X Y}} \equiv \mu
$$

This pair of strategies constitutes the unique equilibrium in the connection continuation game:

\section{Proposition 4}

(i) Suppose either that (a) one or both networks require exclusivity from both sides of the market; or (b) one network requires exclusivity of $A$ and the other requires exclusivity of $B$. Then the continuation game is equivalent to the game when both networks stipulate network routing and its equilibrium is described by Proposition 2.

(ii) Suppose that one or both networks requires exclusivity solely from $A$ and neither network requires exclusivity from $B$. Then the resulting continuation game has a unique equilibrium in which

(a) B joins any network for which $s_{z B}>0$; and joins no network for which $s_{z B} \leq 0$; and 
(b) A joins network $X$ if $s_{X A}>\max \left\{0, \frac{1}{\mu} s_{Y A}\right\}$; joins network $Y$ if $s_{Y A}>\max \left\{0, \mu s_{X A}\right\}$; and joins neither network otherwise (i.e., if $s_{h A} \leq 0$ ); where $\mu$ is defined by expression (11) above.

Because, in (ii), $B$ can be on both networks, there is greater trade than there would be under full exclusivity at a given set of prices.

\section{Network Pricing}

The analysis in the previous section, particularly Proposition 4 Part (i), revealed that there are only five possible continuation games:

(a) Both networks give one side ( $A$ or $B$ ) the right to set the routing rule (see Proposition 1).

(b) One network gives $A$ the right to set the routing rule and the other gives $B$ that right.

(c) Both networks stipulate network routing (see Proposition 2).

(d) One network stipulates network routing and the other chooses one-side-chooses routing (see Proposition 3).

(e) One or both networks requires exclusivity of one side of the market and neither network requires exclusivity of the other side of the market (see Proposition 4, part (ii)).

As this list makes clear, all but game (b) have been analyzed. We, recall, ruled out game (b) because it was unclear how the conflicting routing regimes could be reconciled. In what follows, we assume that, were the networks to have conflicting regimes, their payoffs would be so low because of the resulting conflict (e.g., litigation costs) that they would rationally avoid conflicting routing regimes.

To determine what is the equilibrium configuration of routing regimes, we first need to determine the networks' expected profits in the relevant four continuation games. That is, we must solve for the equilibrium of the pricing game given routing regimes in place. We focus on pure-strategy equilibria. 
Although the setup of the model is straightforward, determining the equilibria of the pricing games is made difficult by the fact that the equilibria of the continuation games depend on the distribution functions, which have heretofore been arbitrary. Even if functional forms are assumed for the distribution functions, calculation of analytic, closed-form equilibrium demands in the continuation games is difficult. Hence, in what follows, we have employed Mathematica to calculate the pricing-game equilibria for specific distributions. ${ }^{21}$

We now assume that the distributions are the uniform distribution on the unit square, $[0,1] \times[0,1]$, and the per-transaction cost lies between 0 and 2 . Table 1 reports the equilibrium profits for various values of the marginal cost, $c$.

In an $A$-chooses routing regime, there is a symmetric Nash equilibrium of the pricing game (i.e., $p_{X A}=p_{Y A} \equiv p_{A}$ and $p_{X B}=p_{Y B} \equiv p_{B}$ ). As shown in the Appendix (Table 3), $p_{A}>p_{B}$, although the difference in the two prices decreases as the transaction cost, $c$, increases. Intuitively, not having the routing choice makes $B$ 's network membership decision more price sensitive ceteris paribus; hence competition for $B$ is fiercer, which leads $B$ to be charged less in equilibrium.

When $X$ is the only network to stipulate network routing, there is an asymmetric Nash equilibrium of the pricing game in which the networks set different prices, but each network sets the same price to both sides of the market (i.e., $p_{X A}=p_{X B} \equiv p_{X}$ and $p_{Y A}=p_{Y B} \equiv p_{Y}$; but $\left.p_{X} \neq p_{Y}\right)$. For the games in which transaction cost, $c$, is low $(c<.35), p_{X}>p_{Y}$. But for games with high transaction costs $(c \geq .35), p_{X}<p_{Y}$. (See Appendix.) Regardless of transaction costs, the network without a network-routing requirement earns greater expected profits than does the network with the network-routing requirement. As Corollary 1 suggests, $X$ carries less traffic in expectation than does $Y$, which translates into less expected profits for $X$.

The game in which both networks stipulate network routing does not have a pure-strategy equilibrium in the pricing game when transaction costs are low (i.e., $c<1$ ). The reason is that, when prices are low and similar, the connection continuation game has multiple equilibria. For instance, if $p_{z k}=1 / 4$ for all $z$ and $k$, then the connection continuation game

${ }^{21}$ The Mathematica program used here and below can be downloaded from: http://faculty.haas.berkeley. edu/hermalin/. 


\begin{tabular}{|c|c|c|c|c|c|}
\hline & $A$-routing & \multicolumn{2}{|c|}{$X$ only network routing } & Both network route & 1-side exclusive \\
\hline$c$ & profit $\equiv \Pi_{A}$ & $\operatorname{profit}_{X} \equiv \Pi_{N O}$ & $\operatorname{profit}_{Y} \equiv \Pi_{A O}$ & profit $\equiv \Pi_{N}$ & profit $\equiv \Pi_{E}$ \\
\hline 0 & .200 & .172 & .222 & \multirow{20}{*}{$\begin{array}{c}\text { No } \\
\text { Pure-Strategy } \\
\text { Equilibrium }\end{array}$} & .170 \\
\hline .05 & .190 & .166 & .208 & & .162 \\
\hline .10 & .180 & .160 & .195 & & .153 \\
\hline .15 & .170 & .153 & .182 & & .144 \\
\hline .20 & .160 & .145 & .169 & & .135 \\
\hline .25 & .150 & .137 & .157 & & .127 \\
\hline .30 & .140 & .129 & .146 & & .119 \\
\hline .35 & .130 & .121 & .135 & & .111 \\
\hline .40 & .121 & .113 & .124 & & .103 \\
\hline .45 & .112 & .105 & .114 & & .095 \\
\hline .50 & .103 & .097 & .106 & & .088 \\
\hline .55 & .094 & .090 & .095 & & .080 \\
\hline .60 & .086 & .082 & .087 & & .074 \\
\hline .65 & .078 & .075 & .079 & & .067 \\
\hline .70 & .070 & .068 & .071 & & .061 \\
\hline .75 & .063 & .061 & .064 & & .055 \\
\hline .80 & .057 & .055 & .057 & & .049 \\
\hline .85 & .050 & .049 & .050 & & .044 \\
\hline .90 & .045 & .043 & .044 & & .039 \\
\hline .95 & .039 & .038 & .039 & & .034 \\
\hline 1.00 & $.341 \times 10^{-1}$ & $.334 \times 10^{-1}$ & $.340 \times 10^{-1}$ & $.196 \times 10^{-1}$ & $.301 \times 10^{-1}$ \\
\hline 1.05 & $.295 \times 10^{-1}$ & $.289 \times 10^{-1}$ & $.293 \times 10^{-1}$ & $.181 \times 10^{-1}$ & $.261 \times 10^{-1}$ \\
\hline 1.10 & $.252 \times 10^{-1}$ & $.248 \times 10^{-1}$ & $.251 \times 10^{-1}$ & $.164 \times 10^{-1}$ & $.225 \times 10^{-1}$ \\
\hline 1.15 & $.214 \times 10^{-1}$ & $.211 \times 10^{-1}$ & $.213 \times 10^{-1}$ & $.145 \times 10^{-1}$ & $.192 \times 10^{-1}$ \\
\hline 1.20 & $.180 \times 10^{-1}$ & $.177 \times 10^{-1}$ & $.179 \times 10^{-1}$ & $.127 \times 10^{-1}$ & $.162 \times 10^{-1}$ \\
\hline 1.25 & $.149 \times 10^{-1}$ & $.147 \times 10^{-1}$ & $.148 \times 10^{-1}$ & $.109 \times 10^{-1}$ & $.135 \times 10^{-1}$ \\
\hline 1.30 & $.122 \times 10^{-1}$ & $.121 \times 10^{-1}$ & $.121 \times 10^{-1}$ & $.918 \times 10^{-2}$ & $.111 \times 10^{-1}$ \\
\hline 1.35 & $.982 \times 10^{-2}$ & $.973 \times 10^{-2}$ & $.978 \times 10^{-2}$ & $.760 \times 10^{-2}$ & $.899 \times 10^{-2}$ \\
\hline 1.40 & $.777 \times 10^{-2}$ & $.771 \times 10^{-2}$ & $.773 \times 10^{-2}$ & $.617 \times 10^{-2}$ & $.714 \times 10^{-2}$ \\
\hline 1.45 & $.601 \times 10^{-2}$ & $.597 \times 10^{-2}$ & $.599 \times 10^{-2}$ & $.489 \times 10^{-2}$ & $.556 \times 10^{-2}$ \\
\hline 1.50 & $.454 \times 10^{-2}$ & $.451 \times 10^{-2}$ & $.452 \times 10^{-2}$ & $.378 \times 10^{-2}$ & $.422 \times 10^{-2}$ \\
\hline 1.55 & $.332 \times 10^{-2}$ & $.330 \times 10^{-2}$ & $.331 \times 10^{-2}$ & $.282 \times 10^{-2}$ & $.311 \times 10^{-2}$ \\
\hline 1.60 & $.234 \times 10^{-2}$ & $.233 \times 10^{-2}$ & $.233 \times 10^{-2}$ & $.203 \times 10^{-2}$ & $.221 \times 10^{-2}$ \\
\hline 1.65 & $.157 \times 10^{-2}$ & $.157 \times 10^{-2}$ & $.157 \times 10^{-2}$ & $.139 \times 10^{-2}$ & $.149 \times 10^{-2}$ \\
\hline 1.70 & $.993 \times 10^{-3}$ & $.990 \times 10^{-3}$ & $.991 \times 10^{-3}$ & $.893 \times 10^{-3}$ & $.948 \times 10^{-3}$ \\
\hline 1.75 & $.576 \times 10^{-3}$ & $.575 \times 10^{-3}$ & $.575 \times 10^{-3}$ & $.528 \times 10^{-3}$ & $.554 \times 10^{-3}$ \\
\hline 1.80 & $.295 \times 10^{-3}$ & $.295 \times 10^{-3}$ & $.295 \times 10^{-3}$ & $.276 \times 10^{-3}$ & $.286 \times 10^{-3}$ \\
\hline 1.85 & $.125 \times 10^{-3}$ & $.125 \times 10^{-3}$ & $.125 \times 10^{-3}$ & $.119 \times 10^{-3}$ & $.122 \times 10^{-3}$ \\
\hline 1.90 & $.370 \times 10^{-4}$ & $.370 \times 10^{-4}$ & $.370 \times 10^{-4}$ & $.358 \times 10^{-4}$ & $.364 \times 10^{-4}$ \\
\hline 1.95 & $.463 \times 10^{-5}$ & $.463 \times 10^{-5}$ & $.463 \times 10^{-5}$ & $.455 \times 10^{-5}$ & $.459 \times 10^{-5}$ \\
\hline
\end{tabular}

Table 1: Comparison of Equilibrium Profits. 
has three equilibria:

1. $\tan \left(\theta_{A}\right)=\tan \left(\theta_{B}\right)=2 / 3$;

2. $\tan \left(\theta_{A}\right)=\tan \left(\theta_{B}\right)=1 ;$ and

3. $\tan \left(\theta_{A}\right)=\tan \left(\theta_{B}\right)=3 / 2$.

In terms of Figure 3, they correspond, respectively, to equilibria that favor $Y$ (i.e., $\overrightarrow{O^{\prime} U}$ closer to horizontal); are neutral (i.e., $\overrightarrow{O^{\prime} U}$ has slope 1); and that favor $X$ (i.e., $\overrightarrow{O^{\prime} U}$ closer to vertical). Moreover, the middle equilibrium (equal market shares) is unstable. Consequently, small changes in price can lead to large changes in market share. This tension, in essence, puts pressures on both networks to undercut on price. However, were prices too low, a network would rationally "stop competing" and seek rents from its loyal consumers. But if a network were to exploit its loyal customers, the other network's best response would be to raise prices, starting the "cycle" over.

For high enough transaction costs $(c \geq 1)$, a symmetric pure-strategy equilibrium exists for the pricing game when both networks stipulate network routing. Both networks charge the same price to both sides of the market (i.e., $p_{z k} \equiv p$ for all $z$ and $k$ ). When prices are high enough, there is a unique pure-strategy equilibrium of the kind described in Proposition 2. The pricing game then becomes a well-behaved game between differentiated duopolists. Because of exclusivity, competition is fiercer between the networks than in the other routing regimes. This and the lower volume of trade result in much lower profits when both networks stipulate network routing than in the other continuation games.

The one-side exclusivity game results in a symmetric equilibrium: $p_{X A}=p_{Y A} \equiv p_{A}$ and $p_{X B}=p_{Y B}=p_{B}$. Moreover, $p_{A}$ and $p_{B}$ tend to be nearly equal.

\section{The Choice of Routing Regime}

Using Table 1, we can solve for the choice of routing regime when the distributions are uniform and $c \geq 1$. Figure 9 presents the routing-regime game in normal form. 


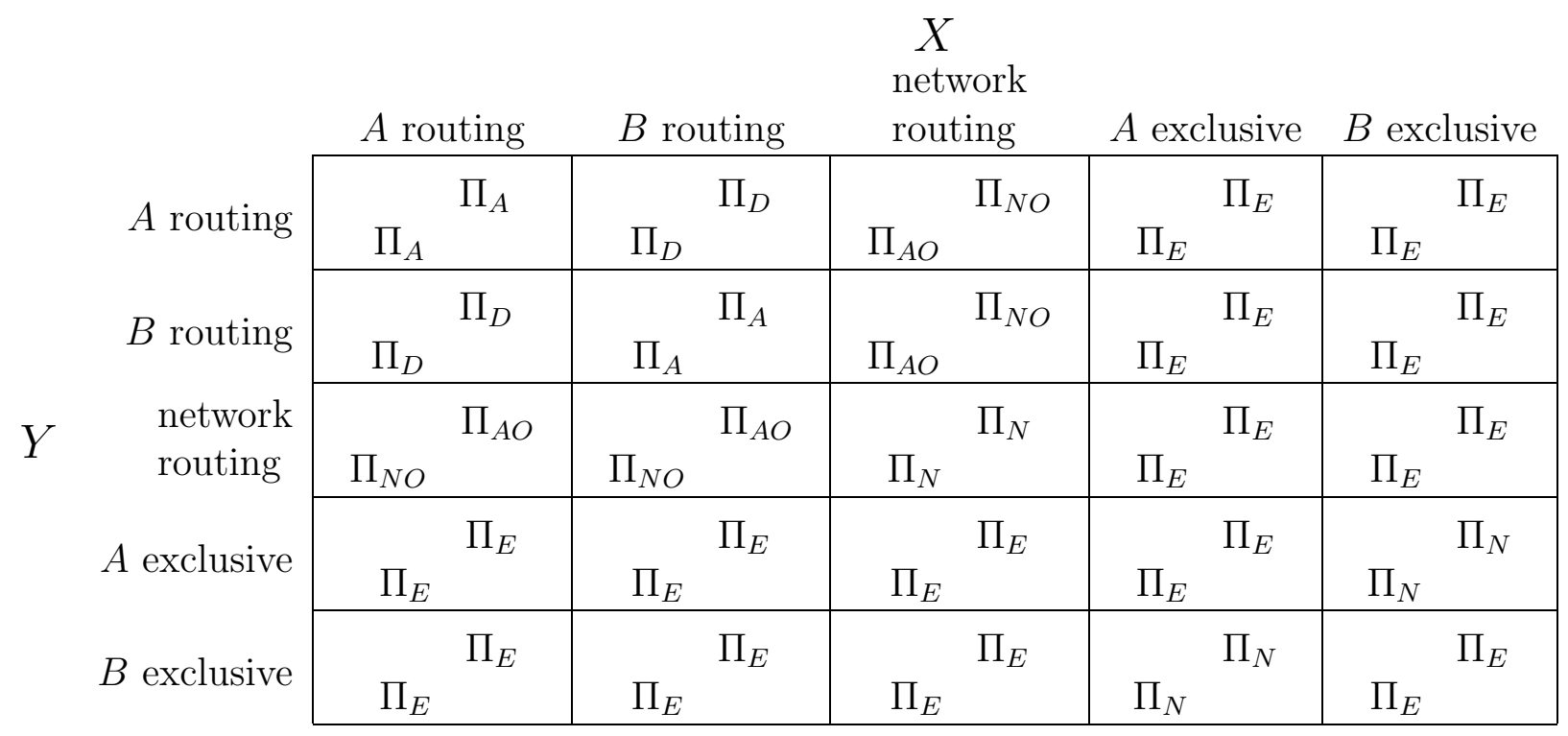

Where

$\Pi_{A}=$ expected profit when both networks have $A$ routing (or both have $B$ routing) ${ }^{a}$

$\Pi_{A O}=$ expected profit for network with $A(B)$ routing when other network has network routing

$\Pi_{N O}=$ expected profit for network with network routing when other network has $A(B)$ routing

$\Pi_{N}=$ expected profit when both networks have network routing ${ }^{b}$

$\Pi_{E}=$ expected profit under one-sided exclusivity ${ }^{c}$

$\Pi_{D}=$ expected profit if networks impose incompatible routing regimes

${ }^{a}$ The assumption of a uniform distribution on the unit square ensures that the continuation game with $A$ routing yields the same profits as the continuation game with $B$ routing. Were the two sides to have different distributions, then this would not hold generally.

${ }^{b}$ Alternatively, one or both networks require exclusivity of both sides of the market; or one network requires exclusivity from $A$, while the other requires exclusivity from $B$. See Proposition 4 .

${ }^{c}$ Specifically, one network (at least) requires exclusivity of one side and neither network requires exclusivity of the other side.

Figure 9: Routing-regime Game. 
From Table 1, we have

$$
\begin{aligned}
\Pi_{A} & >\Pi_{N O}, \\
\Pi_{A} & >\Pi_{E}, \\
\Pi_{A O} & >\Pi_{E}, \\
\Pi_{A O} & >\Pi_{N}, \text { and } \\
\Pi_{E} & >\Pi_{N} ;
\end{aligned}
$$

moreover, by assumption, we have

$$
\Pi_{A}>\Pi_{D}
$$

Inspection of Table 1 establishes the following result.

Proposition 5 Suppose that net consumption benefits are distributed uniformly on $[0,1]^{2}$ and the transaction cost, $c$, exceeds 1. Then the pure-strategy equilibria of the routing-regime game are:

(i) Both networks delegate routing to a common side of the market, either $A$ or $B$.

(ii) Both networks require exclusivity of a common side of the market, but not of the other side.

The networks Pareto prefer an equilibrium in which one side is delegated the authority to choose routing to an equilibrium with one-sided exclusivity. ${ }^{22}$

As indicated above, both trade inefficiency and routing inefficiency can arise under either a one-side-chooses or one-sided-exclusivity routing regime. In the remainder of this section, we compare the resulting levels of total surplus under the two possible equilibrium routing

\footnotetext{
${ }^{22}$ Although the uniform-distribution version of our model predicts that networks should adopt issuer routing (or, possibly, merchant routing), some debit networks actually use network routing. At least in the United States, network routing may be a holdover from the evolution of automatic teller machine networksfor which network routing was prevalent-into point-of-sale debit networks. Moreover, in recent years, an increasing number of networks have implemented issuer routing.
} 
regimes with each other and with the first best. Throughout, we assume independent uniform distributions for $a_{X}, a_{Y}, b_{X}$, and $b_{Y}$.

The first-best level of expected welfare can be calculated by defining $g_{z}=a_{z}+b_{z}$ as total gross consumption benefits if trade occurs on network $z$. Because $a_{z}$ and $b_{z}$ are independently distributed uniformly on $[0,1], g_{z}$ has the following density and cumulative distribution functions, respectively:

$$
\psi(g)=\left\{\begin{array}{l}
g, \text { if } 0 \leq g \leq 1 \\
2-g, \text { if } 1<g \leq 2
\end{array} \quad \text { and } \quad \Psi(g)=\left\{\begin{array}{l}
g^{2} / 2, \text { if } 0 \leq g \leq 1 \\
2 g-g^{2} / 2-1, \text { if } 1<g \leq 2
\end{array} .\right.\right.
$$

Expected welfare is

$$
\mathbb{E} W^{F B}=\int_{0}^{2} \int_{0}^{2} \max \left\{0, g_{X}-c, g_{Y}-c\right\} \psi\left(g_{X}\right) \psi\left(g_{Y}\right) d g_{X} d g_{Y}=2 \int_{c}^{2}(g-c) \Psi(g) \psi(g) d g .
$$

Hence, via tedious calculations, we have

$$
\mathbb{E} W^{F B}=\left\{\begin{array}{l}
\frac{1}{60}\left(74-60 c+3 c^{5}\right), \text { if } 0 \leq c \leq 1 \\
\frac{1}{60}(2-c)^{3}\left(8+12 c-3 c^{2}\right), \text { if } 1<c \leq 2
\end{array} .\right.
$$

For $A$-chooses routing, we have

$$
\begin{aligned}
\mathbb{E} W^{A}= & 2 \alpha_{z}\left(\beta_{z}+\beta_{X Y}\right)\left(\mathbb{E}\left\{a_{z} \mid \text { only on } z\right\}+\mathbb{E}\left\{b_{z} \mid \text { able to trade on } z\right\}-c\right) \\
& +2 \alpha_{X Y} \lambda_{z}\left(\beta_{z}+\beta_{X Y}\right)\left(\mathbb{E}\left\{a_{z} \mid \text { on both, prefers } z\right\}+\mathbb{E}\left\{b_{z} \mid \text { able to trade on } z\right\}-c\right) \\
& +2 \alpha_{X Y}\left(1-\lambda_{z}\right) \beta_{z}\left(\mathbb{E}\left\{a_{z} \mid \text { on both, doesn't prefer } z\right\}+\mathbb{E}\left\{b_{z} \mid \text { only on } z\right\}-c\right),
\end{aligned}
$$

where the factor of 2 reflects that trade is equally likely on either network in the symmetric equilibrium of the pricing game.

For $A$-exclusivity routing, we have

$$
\mathbb{E} W^{E}=2 \tilde{\alpha}_{z}\left(\tilde{\beta}_{z}+\tilde{\beta}_{X Y}\right)\left(\mathbb{E}\left\{a_{z} \mid \text { only on } z\right\}+\mathbb{E}\left\{b_{z} \mid \text { able to trade on } z\right\}-c\right),
$$

where, again, the factor of 2 reflects that trade is equally likely on either network in the symmetric equilibrium of the pricing game and the tildes are a reminder that the probabilities are different than in a one-side-chooses regime.

Table 2 reports expected welfare for the two possible types of equilibria in Proposition 5, as well as the first-best level of expected welfare for comparison. 


\begin{tabular}{|r|r|r|r|}
\hline$c$ & $\begin{array}{c}\text { First best } \\
\mathbb{E} W^{F B}\end{array}$ & $\begin{array}{c}\text { A-routing } \\
\mathbb{E} W^{A}\end{array}$ & $\begin{array}{c}\text { 1-side exclusive } \\
\mathbb{E} W^{E}\end{array}$ \\
\hline \hline 0 & 1.233 & .9477 & .9484 \\
\hline .05 & 1.183 & .8885 & .8868 \\
\hline .10 & 1.133 & .8310 & .8274 \\
\hline .15 & 1.083 & .7753 & .7701 \\
\hline .20 & 1.033 & .7215 & .7151 \\
\hline .25 & .9834 & .6697 & .6623 \\
\hline .30 & .9335 & .6199 & .6118 \\
\hline .35 & .8836 & .5722 & .5635 \\
\hline .40 & .8338 & .5265 & .5176 \\
\hline .45 & .7843 & .4828 & .4739 \\
\hline .50 & .7349 & .4413 & .4324 \\
\hline .55 & .6859 & .4019 & .3932 \\
\hline .60 & .6372 & .3646 & .3562 \\
\hline .65 & .5891 & .3294 & .3214 \\
\hline .70 & .5417 & .2963 & .2888 \\
\hline .75 & .4952 & .2653 & .2583 \\
\hline .80 & .4497 & .2363 & .2299 \\
\hline .85 & .4055 & .2093 & .2035 \\
\hline .90 & .3629 & .1843 & .1791 \\
\hline .95 & .3220 & .1613 & .1566 \\
\hline 1.00 & .2833 & .1401 & .1360 \\
\hline 1.05 & .2471 & .1208 & .1172 \\
\hline 1.10 & .2135 & .1032 & .1002 \\
\hline 1.15 & .1825 & $.8738 \times 10^{-1}$ & $.8481 \times 10^{-1}$ \\
\hline 1.20 & .1543 & $.7318 \times 10^{-1}$ & $.7105 \times 10^{-1}$ \\
\hline 1.25 & .1288 & $.6056 \times 10^{-1}$ & $.5882 \times 10^{-1}$ \\
\hline 1.30 & .1059 & $.4944 \times 10^{-1}$ & $.4805 \times 10^{-1}$ \\
\hline 1.35 & $.8574 \times 10^{-1}$ & $.3973 \times 10^{-1}$ & $.3865 \times 10^{-1}$ \\
\hline 1.40 & $.6811 \times 10^{-1}$ & $.3136 \times 10^{-1}$ & $.3053 \times 10^{-1}$ \\
\hline 1.45 & $.5294 \times 10^{-1}$ & $.2423 \times 10^{-1}$ & $.2362 \times 10^{-1}$ \\
\hline 1.50 & $.4010 \times 10^{-1}$ & $.1826 \times 10^{-1}$ & $.1782 \times 10^{-1}$ \\
\hline 1.55 & $.2945 \times 10^{-1}$ & $.1335 \times 10^{-1}$ & $.1305 \times 10^{-1}$ \\
\hline 1.60 & $.2082 \times 10^{-1}$ & $.9396 \times 10^{-2}$ & $.9201 \times 10^{-2}$ \\
\hline 1.65 & $.1403 \times 10^{-1}$ & $.6308 \times 10^{-2}$ & $.6189 \times 10^{-2}$ \\
\hline 1.70 & $.8879 \times 10^{-2}$ & $.3980 \times 10^{-2}$ & $.3913 \times 10^{-2}$ \\
\hline 1.75 & $.5160 \times 10^{-2}$ & $.2307 \times 10^{-2}$ & $.2273 \times 10^{-2}$ \\
\hline 1.80 & $.2651 \times 10^{-2}$ & $.1182 \times 10^{-2}$ & $.1168 \times 10^{-2}$ \\
\hline 1.85 & $.1121 \times 10^{-2}$ & $.4994 \times 10^{-3}$ & $.4947 \times 10^{-3}$ \\
\hline 1.90 & $.3328 \times 10^{-3}$ & $.1481 \times 10^{-3}$ & $.1464 \times 10^{-3}$ \\
\hline 1.95 & $.4165 \times 10^{-4}$ & $.1852 \times 10^{-4}$ & $.1836 \times 10^{-4}$ \\
\hline & & & \\
\hline
\end{tabular}

Table 2: Comparison of Expected Welfare. 
As seen in Table 2, expected welfare is greater in a one-side-chooses equilibrium than in a one-side-exclusivity equilibrium, except at the lowest cost, $c=0$ (i.e., $\mathbb{E} W^{A}>\mathbb{E} W^{E}$ except for $c=0$ ). Hence, the equilibrium preferred by the networks (see Proposition 5) is also the expected-welfare-maximizing equilibrium.

\section{Extensions}

We next consider four extensions of our baseline model.

\subsection{Video Games and Operating Systems}

Video games are another example of a two-sided market, in which game-playing consumers are on one side and game developers are on the other, with the game console manufacturer serving as the network provider. We can model video game networks as a situation in which technology imposes exclusivity on the consumer side of the market; that is, while a consumer could have two different game systems, it is sufficiently costly that few do in practice. ${ }^{23}$

Video game networks thus play a version of the routing-regime game in Figure 9 in which, essentially, various rows and columns have been eliminated. If we consider $A$ to be the consumer and $B$ to be the game developer, then $A$ is exclusively on one network. If either network or both require exclusivity of $B$, then, as discussed above, the continuation game is equivalent to the game in which both networks stipulate network routing (i.e., the middle row and column in Figure 9). If neither network requires exclusivity of $B$, then the continuation game is equivalent to the $A$-exclusivity (one-side-exclusivity) game. Figure 10 shows the "pruned" routing-choice game. Because $\Pi_{E}>\Pi_{N}$ for the uniform-distribution case, the Pareto-preferred equilibrium of this two-by-two game is for the networks to allow the developers $(B)$ to write for both networks. It is, however, also an equilibrium for both console makers to impose developer exclusivity even in this symmetric case.

A similar analysis can be applied to computer operating systems and application software.

\footnotetext{
${ }^{23}$ There are also costs for the consumer of switching between networks (e.g., unscrewing the connections for one game console from the TV to attach the other). Although it is conceivable that networks could price to make having two game consoles affordable - and such a strategic option may, thus, warrant future attention - it is, nonetheless, an empirical fact that they don't.
} 


\begin{tabular}{|c|c|c|}
\hline & & \\
\hline & $A$ exclusive & $A \& B$ exclusive $^{a}$ \\
\hline$A$ exclusive & $\Pi_{E} \Pi_{E}$ & $\Pi_{N} \Pi_{N}$ \\
\hline$A \& B$ exclusive $^{a}$ & $\Pi_{N} \Pi_{N}$ & $\Pi_{N} \Pi_{N}$ \\
\hline
\end{tabular}

Figure 10: Routing-regime Game for Video Games and Application Software.

${ }^{a}$ Equivalent to network routing

\subsection{The Deciding Party Pays}

It is of some practical interest to consider the effects of a rule requiring that, if one side of the market gets to choose the network, then the other side of the market faces a price of zero. In a different context, this rule has been applied to mobile telephony. Specifically, federal regulators in the United States do not allow calling-party-pays billing when the receiver is a mobile user. In large part, the rationale for this prohibition is that the called party is the one who has made the choice among wireless carriers. ${ }^{24}$ As has been well established by the literature on pricing in two-sided markets, it generally is not efficient to set the price paid by one side of the market to $0 .{ }^{25}$ Moreover, a requirement that only the party with routing control be the one to pay for the service can induce networks to choose a different routing regime than they would under a policy of laissez faire.

The potential adverse profit and welfare effects of a chooser-pays rule are readily seen by considering the following limiting case. Suppose regulation requires that, if network $z$ implements an $A$-chooses routing rule, then it must set $p_{z B}=0$. Observe that, if $c$ exceeds the maximal value of $A$ 's willingness to pay, then the profits associated with letting $A$ choose the network are zero because there is no trade. A regime under which the costs of a transaction can be shared between the two parties may, however, allow profitable trade to

\footnotetext{
${ }^{24}$ Observe, however, that, if the calling party is familiar with the called party's telephone numbers, then one could just as well prohibit called-party-pays billing on the grounds that the calling party chooses the carrier (routing) by deciding to dial the wireless number instead of the wireline one.

${ }^{25}$ See, for example, Jeon et al. (2003), Kim and Lim (2001), and Hermalin and Katz (2004). Doyle and Smith (1998) focus on the issue with particular regard to mobile telephony.
} 
occur. Specifically, consider our case in which user types are uniformly distributed on the unit square. As $c$ goes to one, the profits associated with either issuer or merchant routing go to zero, but other routing regimes remain profitable.

Straightforward computations show that, for all $c \in(1-\delta, 2)$, for some $\delta>0, \Pi_{A}<\Pi_{N O}$. Moreover, for all $c \in(1,2), \Pi_{A O}<\Pi_{E}$.

Proposition 6 Suppose that net consumption benefits are distributed uniformly on $[0,1]^{2}$ and the transaction cost, $c$, is in $(1,2)$. Then the pure-strategy equilibria of the routingregime game with a chooser-pays regulatory requirement are:

(i) One network chooses network routing and the other network chooses either A exclusivity or $B$ exclusivity.

(ii) Both networks require exclusivity of a common side of the market, but not of the other side.

These equilibria all have the same connection continuation game and give rise to identical outcomes in terms of equilibrium prices, transactions quantities, profit, and welfare.

Coupled with Proposition 6, the rankings in Table 2 imply the following:

Corollary 2 A chooser-pays regulation results in lower equilibrium profits and welfare relative to the networks' Pareto-preferred equilibrium absent a chooser-pays requirement.

\subsection{A Hotelling Model}

Many analyses in the two-sided markets literature have employed Hotelling models of product differentiation (e.g., Rochet and Tirole, 2003). A Hotelling model can be considered a special case of our more general model. Specifically, let the gross consumption benefits be $(a, 1-a)$ and $(b, 1-b)$ for $A$ and $B$, respectively, where $a \sim \mathrm{U}[0,1]$ and $b \sim \mathrm{U}[0,1]$. In terms of the spatial metaphor, there are unit transportion costs in a linear city in which $X$ has address 1 , $Y$ has address 0 , and users on both sides of the market are uniformly distributed across the city. 
In what follows, it is useful to observe that, by Lemma 1 , if $\min \left\{p_{X k}, p_{Y k}\right\} \geq 1 / 2$, then user $k$ will never elect to be on both networks.

Suppose that either $A$ has the choice of routing rule or $B$ is required to be exclusively on one network, while $A$ is not. The transaction volume on network $X$ is

$$
D_{X}=\alpha_{X}\left(\beta_{X}+\beta_{X Y}\right)+\alpha_{X Y}\left(\beta_{X}+\lambda_{X} \beta_{X Y}\right)
$$

Uniformity implies $\lambda_{X}=\lambda_{Y}=1 / 2$, so

$$
D_{X}=\left(\alpha_{X}+\alpha_{X Y}\right) \beta_{X}+\beta_{X Y}\left(\alpha_{X}+\alpha_{X Y} / 2\right)
$$

thus, substituting for $\alpha_{X}$ and $\alpha_{X Y}$,

$$
D_{X}(\mathbf{p})=\left(1-p_{X A}\right) \beta_{X}(\mathbf{p})+\left(\frac{1}{2}\left(1-p_{X A}-p_{Y A}\right)+p_{Y A}\right) \beta_{X Y}(\mathbf{p}) .
$$

where $\mathbf{p} \equiv\left(p_{X A}, p_{X B}, p_{Y A}, p_{Y B}\right)$.

Consider the case in which $B$ is required to be exclusive (so $\beta_{X Y} \equiv 0$ ). We will show there cannot be a symmetric equilibrium in which both $p_{B}<1 / 2$ and $p_{A}<1 / 2$. Suppose not and write $X$ 's profits if it deviates from the candidate symmetric equilibrium by $\varepsilon$ :

$$
\Pi_{X}=\left(p_{A}+p_{B}+\varepsilon-c\right) D_{X}\left(p_{A}, p_{B}+\varepsilon, p_{A}, p_{B}\right)
$$

which, substituting for $\beta_{X}$ in equation (12) and using the result in equation (13),

$$
=\left(p_{A}+\varepsilon+p_{B}-c\right)\left(1-p_{A}\right) \frac{1-p_{B}-\varepsilon+p_{B}}{2} .
$$

Because the networks are in equilibrium, it must be that $\partial \Pi_{X} / \partial \varepsilon_{\mid \varepsilon=0}=0$. But

$$
\begin{aligned}
{\frac{\partial \prod_{X}}{\partial \varepsilon_{\mid \varepsilon=0}}} & \left(1-p_{A}\right)\left(1-\left(p_{A}+p_{B}-c\right)\right) \\
& >0
\end{aligned}
$$

if, as assumed, $p_{A}+p_{B}<1$. By contradiction, we have established that, if a symmetric equilibrium exists, $p_{k} \geq 1 / 2$ for users on at least one side of the market. Numerical calculations verify that $p_{B}=1 / 2$ and $p_{A}=1 / 4+c / 2$ is a symmetric equilibrium for $c \leq 1 / 2$. If $c>1 / 2$, then $p_{A}=p_{B}=(1+c) / 3$. 
A similar analysis is possible for the case in which $A$ chooses the network when both are available for a given transaction. The price to $B$ can never be less than $1 / 2$ in a symmetric equilibrium. Moreover, the equilibrium prices are the same: $p_{B}=1 / 2$ and $p_{A}=1 / 4+c / 2$ if $c \leq 1 / 2$; and $p_{A}=p_{B}=(1+c) / 3$ if $c>1 / 2$. Finally, because $B$ is never on both networks, the probabilities of trade are the same as in the $B$-exclusive case; hence, profits and welfare are the same.

Now consider the pricing game when $X$ has stipulated network routing and $Y$ permits either issuer or merchant routing. Because of a user's obligation under $X$ 's network routing stipulation, being assigned the routing choice by $Y$ is worthless. Hence, $A$ and $B$ are effectively in symmetric positions. If, however, $c \leq 1 / 2$, then this initial symmetry is broken in equilibrium; there are two asymmetric pure-strategy equilibria of the pricing game. In one, $p_{A}=1 / 4+c / 2$ and $p_{B}=1 / 2 ;$ and, in the other, $p_{A}=1 / 2$ and $p_{B}=1 / 4+c / 2$. If $c>1 / 2$, then symmetry is restored: $p_{A}=p_{B}=(1+c) / 3$. Observe, that the equilibria of this pricing game are the same as in the two previously considered pricing games.

As in the previous games, one side, say $B$, is always pushed to be exclusive; that is, $p_{X B}=p_{Y B} \geq 1 / 2$. Hence, from expression (9), $\Omega_{A}=90^{\circ}$ and, thus, from Figure 5 , the probability that $A$ is willing to trade on $z$ is $1-p_{z A}$. It is readily shown that $p_{z A}=1 / 4+c / 2$ is a best response for both $X$ and $Y$ if $c \leq 1 / 2$. If $p_{A}=1 / 4+c / 2, c \leq 1 / 2$, and $p_{z^{\prime} B}=1 / 2$, $z^{\prime} \neq z$, then $B$ 's demand for $z$ is just $1-p_{z B}$ if $p_{z B} \geq 1 / 2$. It is straightforward to prove that $p_{z B}>1 / 2$ then yields lower expected profits for $z$ than $p_{z B}=1 / 2$. Thus, neither network should deviate upward from $p_{B}=1 / 2$. If $Y$ drops its price by $\varepsilon$ from $p_{Y B}=1 / 2$, then, of the $\varepsilon B$ types that $Y$ will induce to join its network, a significant fraction will remain on $X$ as well. Moreover, the small increase in $\beta_{X Y}$ will induce only a small fraction of $A$ 's types to switch from belonging to both to belonging to $Y$ exclusively. It can be shown that the total gain in expected sales for $Y$ is too small to compensate for the drop in $p_{Y B}$; that is, $Y$ doesn't wish to deviate downward. If $X$ drops its price to $B$ by $\varepsilon$ from $1 / 2$, then it gains fewer than $\varepsilon B$ types (some types who would now enjoy positive surplus trading over $X$ prefer to remain solely on $Y$ because $s_{Y B}$ is sufficiently greater than $s_{X B}$ ). It also loses some types of $A$ that were on both networks, because they respond to the increase in $\beta_{X Y}$ by quitting network $X$. Hence, as with $Y$, the limited gain in sales does not compensate for 
the drop in price. Network $X$ therefore doesn't wish to deviate downward from $p_{X B}=1 / 2$ either. For $c>1 / 2$, neither network would find it in its interest to price so low that some types wish to join both networks; that is, the networks are essentially monopolists to their ends of the linear city and $p_{A}=p_{B}=(1+c) / 3$ are readily shown to be the profit-maximizing monopoly prices.

To summarize:

Proposition 7 Suppose that user preferences have been generated by the Hotelling model (i.e., $\mathbf{a}=(a, 1-a), \mathbf{b}=(b, 1-b)$, where $a$ and $b \sim \mathrm{U}[0,1])$. If

- both networks implement $k$-chooses routing; or

- at least one network requires $k^{\prime}, k^{\prime} \neq k$, to be exclusive and neither network requires $k$ to be exclusive; or

- one network adopts network routing and the other adopts either $k$-chooses or $k^{\prime}$-chooses routing;

then the pricing game has the following equilibria:

(i) if $c \leq 1 / 2$, then the equilibrium prices are $p_{X k}=p_{Y k}=1 / 4+c / 2$ and $p_{X k^{\prime}}=p_{Y k^{\prime}}=$ $1 / 2$, for $k=A, B$;

(ii) if $c>1 / 2$, then the equilibrium prices are $p_{X A}=p_{Y A}=p_{X B}=p_{Y B}=(1+c) / 3$.

Equilibrium profits and welfare are the same across the routing regimes.

The intuition for why identical outcomes hold across three different routing regimes (in contrast to the analysis in Section 5) is the now familiar idea that membership decisions can trump formal authority. As shown above, the networks always set prices greater than or equal to $1 / 2$ for at least one side of the market, say $B$. Given such prices, no type of $B$ can have positive surplus levels from both networks at once (in terms of our earlier notation, $s_{\ell B} \leq 0$ for all types of $B$ ). Therefore, $A$ 's choice of network and, thus, the networks' pricing to $A$ have no effect on $B$ 's choice of network. Moreover, given that $B$ makes an exclusive network choice, $A$ has no reason not to join all networks that yield it positive surplus, regardless of 
the routing rules; $B$ 's choice of network faces $A$ with an all-or-nothing choice with regard to which network will handle the transaction if one takes place. Thus, when a network changes its price to $A$, there is no effect on the other network's sales. In this regard, the two networks act as monopolists under all three routing regimes when setting their prices to $A$.

If the networks require exclusivity of both sides, then it can be shown that the equilibrium prices are

$$
p_{X A}=p_{Y A}=p_{X B}=p_{Y B}=\left\{\begin{array}{ll}
1 / 2 & \text { if } c \leq 1 / 2 \\
(1+c) / 3 & \text { if } c>1 / 2
\end{array} .\right.
$$

Because, as noted previously, once one side is exclusive, a network need not care whether members of the other side join the rival network (only whether they join it), it follows that the networks must be better off with the equilibrium pricing in Proposition 7 when $c \leq 1 / 2$ than with the prices in (14). That is, consistent with the results in Section 5, double-sided exclusivity is not the optimal routing regime for the networks (at least when cost is low).

When cost is high enough in a Hotelling model (i.e., $c>1 / 2$ ), then Proposition 7 and expression (14) reveal that routing regime doesn't matter. Once cost is high enough, it is never in a network's interest to price low enough to induce a user to join it when the user intrinsically prefers the other network (e.g., for high $c$, network $X$ will never find it profitable to induce user $A$ to join $X$ if $a_{Y}>a_{X}$ ). Obviously, if no one is ever on both networks, then routing regime is irrelevant.

The congruence of prices and profits in the Hotelling version of our model across routing regimes (the three regimes in Proposition 7 and all four regimes if $c>1 / 2$ ) contrasts with the results when types are uniformly distributed on the unit square (see Tables 1 and 3). This suggests that analyses of Hotelling models of two-sided markets must be viewed in perspective; the high degree of differentiation in such models can lead to extreme outcomes (e.g., here, it results in one side's always being exclusive).

\subsection{Rent Dissipation in the Absence of Product Differentiation}

Our analysis has heretofore been restricted to the case of symmetric user preferences with product differentiation. We now examine equilibrium when users do not perceive intrinsic differences between the two networks. Specifically, we ask whether there exist pure-strategy equilibria in which networks earn positive profits. 
The answer is "no" when there is no product differentiation on either side of the market and at least one side joins any network that offers it positive surplus (i.e., one-side-chooses routing or one-side exclusivity). To see why, suppose, to the contrary, that $p_{X A}^{e}, p_{X B}^{e}, p_{Y A}^{e}$, and $p_{Y B}^{e}$ are equilibrium prices and that at least one of the networks earns positive profits. Let $A$ be the side that is willing to join both networks (i.e., $A$ has the routing choice or $B$ is the only side that must be exclusive). Moreover, label the networks so that $X$ 's profits are at least as large as $Y$ 's. Now, suppose that $Y$ were to deviate from the candidate equilibrium by charging $p_{Y A}^{d}=p_{X A}^{e}-\varepsilon$ and $p_{Y B}^{d}=p_{X B}^{e}-\varepsilon$, where $\varepsilon$ is an arbitrarily small positive number. Then any $A$ on $X$ will also be on $Y$ and $B$ can, thus, rationally choose to be exclusively on $Y$. In equilibrium, all transactions go over network $Y$. It is readily shown that, for sufficiently small choices of $\varepsilon$, the deviation would raise network $Y$ 's profits. Therefore, any prices that yield positive profits cannot constitute a pure strategy equilibrium when there is no intrinsic differentiation of the two networks.

If one network stipulates network routing, while the other does not, then this simple undercutting argument still applies, but is somewhat more involved. Suppose, first that $X$, the network earning weakly greater profits, is the one that stipulated network routing. If $Y$ undercuts as above, then, neither side would join $X$ exclusively. From expression (9), that means that the dividing ray between "join $Y$ only" and "join both" (see Figure 5) has slope one. Because $p_{Y}<p_{X}$, that ray lies below the $45^{\circ}$ line, and everyone who joins a network joins $Y$ only. It follows that $Y$ 's profits are greater from this deviation. Suppose, instead, that $X$ has not stipulated network routing and $Y$, which has, undercuts. Then both $A$ and $B$ should join both networks, and-because network routing stipulates that all traffic go on $Y$ - this would be a profitable deviation for $Y$.

When both networks impose network routing, simple undercutting arguments are insufficient because one must account for how $A$ and $B$ form beliefs about who is on which network following out-of-equilibrium pricing by one of the networks (a point also made by Caillaud and Jullien, 2001, among others). On the other hand, if, as seems reasonable in this context, $A$ and $B$ believe that they should join the network that charges a lower price to both sides, then the same undercutting argument again rules out pure-strategy equilibria in which one or both networks earn positive expected profits. 
When both networks stipulate network routing in a setting without differentiation, there exists an equilibrium in which both networks charge the same prices, $p_{A}$ and $p_{B}=c-p_{A}$, but all traffic is on just one of the networks, say $X$. Indeed, except for the knife-edge case in which each network attracts exactly half of each side, the only pure-strategy equilibria must exhibit this tipping. ${ }^{26}$

It is also interesting to ask what happens when users on one side of the market consider the networks to be differentiated, but users on the other side do not. Suppose, for example, that $A$ 's type is uniformly distributed on the unit square, $[0,1] \times[0,1]$, while $B$ 's type is uniformly distributed on the forty-five degree line from $(0,0)$ to $(1,1)$. Further suppose that the per-transaction cost lies between 0 and 2. A reasonable hypothesis might be that network price competition to attract $B$ would dissipate any profits that might be earned as the result of the differentiation perceived by $A$. This hypothesis need not hold, however, because the networks endogenously differentiate themselves from $B$ 's perspective by differentially attracting the various types of $A$.

Under a $B$-chooses regime, for example, numerical calculations show that the networks charge equal equilibrium prices to the two sides. ${ }^{27}$ That is, even though $B$ is indifferent between the two networks and $B$ is the side that nominally chooses the network, competition is just as strong to attract $A$ as $B$. Moreover, the price is not driven to cost, so that firms earn positive equilibrium profits. Product differentiation on one side of the market is enough to create differentiation on both sides because, even though $B$ does not distinguish different underlying network characteristics, the two networks do offer $B$ access to different sets of user types on the other side of the market. Hence, a small reduction in $p_{z B}$ will not lead to a discontinuous jump in sales for network $z$ because high-value types of $B$ will remain on both networks in order to be able to trade with types of $A$ that have joined only one network.

In an $A$-chooses regime, $A$ pays a higher equilibrium transactions price than does $B .^{28}$ As in the $B$-chooses regime, the sum of the prices charged to the two sides of the market

\footnotetext{
${ }^{26}$ Hermalin and Katz (2004) consider how the cost $c$ should be optimally split between $p_{A}$ and $p_{B}$ in situations such as this.

${ }^{27}$ We assume that $B$ chooses the routing by tossing a fair coin when $p_{X B}=p_{Y B}$.

${ }^{28}$ For other routing rules, no pure-strategy equilibria exist.
} 
exceeds costs, and the networks earn positive equilibrium profits.

\section{Conclusion}

We have examined the choice of routing rules for a set of situations in which two networks compete for business. The principal motivating example is debit-card networks, but other payment networks, communications networks, and hardware-software networks raise similar issues.

The central insight from analysis of the connection continuation game is that membership decisions can override formal rules and lead to counterintuitive equilibrium outcomes. Thus, transactions tend to be routed over $B$ 's preferred network when $A$ is given the formal authority to choose the routing if both sides belong to both networks. Similarly, for some distributions of types, a network that imposes network routing in an otherwise symmetric setting will have a lower market share than its rival. Intuitively, a user who cannot control the routing choice has greater incentives to join solely its preferred network than does a user that can choose among networks on a transaction-by-transaction basis.

Our main result for the overall game establishes conditions under which competing networks should adopt routing rules that give one side of the market control of the routing choice (Proposition 5). Intuitively, such a routing regime serves to get many people on both networks, which lessens the all-or-nothing type of competition that exists with network routing. Moreover, because many people join both networks, the volume of trade is greater (i.e., the likelihood of a trade inefficiency is reduced). This equilibrium is also welfare maximizing relative to the other routing regime, exclusivity, that can be sustained as an equilibrium of the routing-choice game.

Clearly work remains to be done. There are several profitable extensions to consider. One is networks that are asymmetrically positioned in terms of costs, consumer preferences, (e.g., $\operatorname{Pr}\left\{a_{X}>a_{Y}\right\}>1 / 2$ ), or installed base. Intuitively, such cases might be more likely to give rise to equilibria in which the "leading" network imposes an exclusivity or network routing requirement in order to capitalize on its superior position. Second, it would be interesting to consider situations in which there are fixed costs of joining a network or 
networks impose membership fees in addition to per-transactions charges. Lastly, it would be useful to characterize mixed strategy equilibria. 


\section{Appendix}

\begin{tabular}{|c|c|c|c|c|c|c|c|}
\hline \multirow[b]{2}{*}{$c$} & \multicolumn{2}{|c|}{$A$-routing } & \multicolumn{2}{|c|}{$X$ only network routing } & \multirow{2}{*}{$\begin{array}{c}\text { Both network route } \\
p_{z k}\end{array}$} & \multicolumn{2}{|c|}{ 1-side exclusive } \\
\hline & $p_{z A}$ & $p_{z B}$ & $p_{X k}$ & $p_{Y k}$ & & $p_{z A}$ & $p_{z B}$ \\
\hline 0 & .314 & .262 & .285 & .280 & \multirow{20}{*}{$\begin{array}{c}\text { No } \\
\text { Pure-Strategy } \\
\text { Equilibrium }\end{array}$} & .236 & .236 \\
\hline .05 & .334 & .286 & .306 & .302 & & .258 & .258 \\
\hline .10 & .353 & .309 & .327 & .324 & & 280 & .280 \\
\hline .15 & .371 & .332 & .347 & .345 & & .302 & .302 \\
\hline .20 & .389 & .354 & .367 & .366 & & .324 & .324 \\
\hline .25 & .407 & .375 & .387 & .386 & & .345 & .345 \\
\hline .30 & .425 & .397 & .407 & .407 & & .366 & .366 \\
\hline .35 & .442 & .417 & .426 & .426 & & .387 & .387 \\
\hline .40 & .460 & .438 & .445 & .446 & & .408 & .408 \\
\hline .45 & .477 & .458 & .464 & .465 & & .429 & .429 \\
\hline .50 & .494 & .477 & .482 & .484 & & .449 & .449 \\
\hline .55 & .512 & .497 & .501 & .503 & & .470 & .470 \\
\hline .60 & .529 & .516 & .519 & .521 & & .490 & .490 \\
\hline .65 & .546 & .535 & .537 & .540 & & .510 & .510 \\
\hline .70 & .563 & .553 & .555 & .558 & & .530 & .530 \\
\hline .75 & .580 & .572 & .573 & .576 & & .550 & .550 \\
\hline .80 & .597 & .590 & .591 & .593 & & .569 & .569 \\
\hline .85 & .614 & .608 & .609 & .611 & & .588 & .588 \\
\hline .90 & .631 & .626 & .627 & .629 & & .608 & .608 \\
\hline .95 & .648 & .644 & .644 & .646 & & .627 & .627 \\
\hline 1.00 & .665 & .662 & .662 & .663 & .594 & .646 & .645 \\
\hline 1.05 & .682 & .679 & .679 & .681 & .621 & .665 & .664 \\
\hline 1.10 & $\begin{array}{l}.699 \\
\end{array}$ & .696 & .696 & .698 & .647 & .684 & .683 \\
\hline 1.15 & .715 & .714 & .714 & .715 & .671 & .702 & .701 \\
\hline 1.20 & .732 & .731 & .731 & .732 & .695 & .721 & .720 \\
\hline 1.25 & .749 & .748 & .748 & .749 & .717 & .739 & .738 \\
\hline 1.30 & .766 & .765 & .765 & .766 & .739 & .757 & .756 \\
\hline 1.35 & .783 & .782 & .782 & .783 & .761 & .775 & .775 \\
\hline 1.40 & .800 & .800 & .799 & .800 & .781 & .793 & .793 \\
\hline 1.45 & .816 & .816 & .816 & .816 & .802 & .811 & .810 \\
\hline 1.50 & .833 & .833 & .833 & .833 & .821 & .829 & .828 \\
\hline 1.55 & .850 & .850 & .850 & .850 & .841 & .846 & .846 \\
\hline 1.60 & .867 & .866 & .866 & .867 & .859 & .864 & .863 \\
\hline 1.65 & .883 & .883 & .883 & .883 & .880 & .881 & .881 \\
\hline 1.70 & .900 & .900 & .900 & .900 & .898 & .898 & .898 \\
\hline 1.75 & .917 & .917 & .917 & .917 & .914 & .916 & .915 \\
\hline 1.80 & .933 & .933 & .933 & .933 & .932 & .933 & .932 \\
\hline 1.85 & .950 & .950 & .950 & .950 & .949 & .950 & .949 \\
\hline 1.90 & .967 & .967 & .967 & .967 & .966 & .967 & .967 \\
\hline 1.95 & .983 & .983 & .983 & .983 & .983 & .983 & .983 \\
\hline
\end{tabular}

Table 3: Comparison of Equilibrium Prices. 


\section{References}

Armstrong, Mark, "Competition in Two-Sided Markets," January 2004. Working Paper, Department of Economics, University College London.

Caillaud, Bernard and Bruno Jullien, "Competing Cybermediaries," European Economic Review, 2001, 45, 797-808.

_ and _ , "Chicken \& Egg: Competition Among Intermediation Service Providers," RAND Journal of Economics, Summer 2003, 34 (2), 309-328.

Crémer, Jacques, Patrick Rey, and Jean Tirole, "Connectivity in the Commercial Internet," Journal of Industrial Economics, 2000, 48 (4), 433-472.

Doyle, Chris and Jennifer C. Smith, "Market Structure in Mobile Telecoms: Qualified Indirect Access and the Receiver Pays Principle," Information Economics and Policy, December 1998, 10 (4), 471-488.

Guthrie, Graeme and Julian Wright, "Competing Payment Systems," September 2003. Working Paper, National University of Singapore.

Hahn, Jong-Hee, "Nonlinear Pricing of a Telecommunications Service with Call and Network Externalities," International Journal of Industrial Organization, 2003, 21 (7), 949967.

Hermalin, Benjamin E. and Michael L. Katz, "Sender or Receiver: Who Should Pay to Exchange an Electronic Message?," RAND Journal of Economics, 2004. Forthcoming.

Jeon, Doh-Shin, Jean-Jacques Laffont, and Jean Tirole, "On the Receiver Pays Principle," RAND Journal of Economics, 2003. Forthcoming.

Kim, Jeong-You and Yoonsung Lim, "An Economic Analysis of the Receiver Pay Principle," Information Economics and Policy, 2001, 13, 231-260. 
Laffont, Jean-Jacques, Scott Marcus, Patrick Rey, and Jean Tirole, "Internet Interconnection and the Off-Net-Cost Pricing Principle," RAND Journal of Economics, Summer 2003, 34 (2), 370-390.

Rochet, Jean-Charles, "The Theory of Interchange Fees: A Synthesis of Recent Contributions," Review of Network Economics, 2003, 2 (2), 97-124.

_ and Jean Tirole, "Platform Competition in Two-Sided Markets," Journal of the European Economic Association, June 2003, 1 (4), 990-1029.

_ and _ , "Defining Two-Sided Markets," January 2004. Working Paper, IDEI, Toulouse.

Schmalensee, Richard, "Payment Systems and Interchange Fees," Journal of Industrial Economics, 2002, 50 (2), 103-122.

Squire, L., "Some Aspects of Optimal Pricing for Telecommunications," The Bell Journal of Economics and Management Science, Autumn 1973, 4 (2), 515-525.

Srinagesh, Padmanabhan and Jiong Gong, "A Model of Telecommunications Demand with Call Externality," Working Paper, Bellcore 1996.

Tieman, Alexander F. and Wilko Bolt, "Pricing Electronic Payments Services: An IO Approach," Technical Report, Research Department, De Nederlandsche Bank, Amsterdam 2003. 